This item was submitted to Loughborough's Research Repository by the author.

Items in Figshare are protected by copyright, with all rights reserved, unless otherwise indicated.

\title{
A novel zero-watermarking scheme with enhanced distinguishability and robustness for volumetric medical imaging
}

PLEASE CITE THE PUBLISHED VERSION

https://doi.org/10.1016/j.image.2020.116124

PUBLISHER

Elsevier

VERSION

AM (Accepted Manuscript)

\section{PUBLISHER STATEMENT}

This paper was accepted for publication in the journal Signal Processing: Image Communication and the definitive published version is available at https://doi.org/10.1016/j.image.2020.116124

LICENCE

CC BY-NC-ND 4.0

\section{REPOSITORY RECORD}

Liu, Xiyao, Yuying Sun, Jiahui Wang, Cundian Yang, Yayun Zhang, Lei Wang, Yan Chen, and Hui Fang. 2021. "A Novel Zero-watermarking Scheme with Enhanced Distinguishability and Robustness for Volumetric Medical Imaging". Loughborough University. https://hdl.handle.net/2134/13514179.v1. 
- An improved lossless zero-watermarking is proposed for volumetric medical imaging

- Ring statistics are designed to ensure the distinguishability and robustness

- Intra-slice variations are deployed to enhance the watermarking distinguishability

- A logistic-logistic based chaotic system is used to ensure the watermarking security 


\title{
A novel zero-watermarking scheme with enhanced distinguishability and robustness for volumetric medical imaging
}

\author{
Xiyao Liu ${ }^{\mathrm{a}}$, Yuying Sun ${ }^{\mathrm{a}}$, Jiahui Wang ${ }^{\mathrm{b}}$, Cundian Yang ${ }^{\mathrm{a}}$, Yayun Zhang ${ }^{\mathrm{a}}$, Lei \\ Wang $^{\mathrm{a}}$, Yan Chen ${ }^{\mathrm{c}, *}$, Hui Fang ${ }^{\mathrm{d}}$ \\ ${ }^{a}$ School of Computer Science and Engineering, Central South University, Changsha 410083, China \\ ${ }^{b}$ Department of Information and Security, The State Information Center, Beijing 100045, China \\ ${ }^{c}$ School of Medicine, University of Nottingham, Nottingham NG5 1PB, U.K. \\ ${ }^{d}$ Department of Computer Science, Loughborough University, Loughborough LE11 3TU, U.K.
}

\begin{abstract}
The authenticity and copyright protection of volumetric medical images has become extremely important when these images are distributed online for diagnosis and education purpose. Compared to the authenticity and copyright protection of conventional images, there are two additional challenges for protecting the volumetric medical images. On one hand, the content of the protected medical images must be distortion-free to ensure unbiased diagnosis. On the other hand, it requires enhanced distinguishability to avoid misclassification of non-protected images into the protected set because volumetric medical images of different persons in the same modality share similar visual structures. To address these issues, a novel multi-slice feature based zero-watermarking scheme with enhanced distinguishability and robustness for volumetric medical imaging is proposed. In this scheme, ring statistics are deployed to guarantee both the watermarking distinguishability and robustness. In addition, an intra-slice variation based mechanism is designed to further enhance the watermarking distinguishability. Finally, a logistic-logistic system based chaotic map is used to ensure the watermarking security. Our experimental results demonstrate that the proposed scheme not only satisfies the lossless quality requirement but also ensures the watermarking distinguishability and robustness, which outperforms the state-of-the-art schemes.
\end{abstract}

Key words: Volumetric medical imaging, Zero-watermarking, Multi-slice feature, Ring statistic, Intra-slice variation, Chaotic map

\footnotetext{
${ }^{*}$ Corresponding author

Email addresses: lxyzoewx@csu.edu.cn (Xiyao Liu), 184612338@csu.edu.cn (Yuying Sun), wangjiahui@sic.gov.cn (Jiahui Wang), 8208180918@csu.edu.cn (Cundian Yang), 184612335@csu.edu.cn (Yayun Zhang), wanglei@csu.edu.cn (Lei Wang), yan.chen.ac.uk@gmail.com (Yan Chen), H.Fang@lboro.ac.uk (Hui Fang)
} 


\section{Introduction}

Volumetric Medical Imaging (VMI), such as Computed Tomography (CT) and Magnetic Resonance Imaging (MRI), has become an indispensable component in modern diagnostic radiology $[1,2]$. This technology is widely used to diagnose a variety of diseases, e.g., lung cancer [3, 4], chest lesions [5, 6] and cerebral disease $[7,8]$, as it represents the 3D anatomical structure of human body in an intuitive way by scanning and visualizing a sequence of $2 \mathrm{D}$ image slices [9]. In recent years, the number of VMIs that are shared and exchanged across collaborated hospitals and research institutes is increasing significantly due to the popularity of telemedicine, collaborative diagnosis and medical education.

The authenticity and copyright protection of VMIs has become crucial when these images are distributed online for diagnosis and education purpose. There are two additional challenges for protecting the VMIs comparing to the traditional image copyright protection: (1) the content of the protected medical image must be distortion-free to ensure unbiased diagnosis; and (2) it requires enhanced distinguishability to avoid misclassification of non-protected images into the protected ones because VMIs of different persons in the same modality share similar visual structures. Therefore, the motivation of our work is to design a novel watermarking scheme to satisfy these additional requirements.

Three categories of digital watermarking schemes are suitable for authenticity and copyright protection of medical images, which are ROI lossless watermarking [1018]; reversible watermarking [19-29]; and zero-watermarking [30-40]. ROI lossless watermarking schemes embed watermark into medical images while keeping their ROI regions distortion-free to minimize the bias on medical diagnosis. However, it is a complicated and tedious task to segment the ROI and RONI regions accurately. In addition, it is still under argument whether the distortion in RONI regions affects the diagnosis accuracy. Reversible watermarking guarantees the lossless recovery of the original medical imaging after watermarking extraction. However, the robustness of the schemes is relatively weak comparing to non-reversible schemes. Zero-watermarking schemes establish the relationships between image features and the watermark to provide reliable authenticity and copyright protection without any embedding process or imaging distortion. Although they are the most promising methods for protecting the authenticity and copyright of medical images, one major concern of applying the schemes to the volumetric images is the insufficient feature distinguishability between different images. In other words, the false positive of authenticity and copyright identification based on the extracted features is quite high because the images of different persons taken by using the same modality share a lot of common characteristics. As illustrated in Fig. 1, CT or MRI images from different patients have similar visual structures. In addition, the robustness against geometrical attacks, such as flipping and rotations, needs to be ensured at the same time of guaranteeing the distinguishability, which is still a challenge for zero-watermarking schemes because their robustness and distinguishability are always contradictory.

In this paper, we propose a novel zero-watermarking scheme for VMI which not only satisfies the lossless quality requirement but also enhances both the water- 


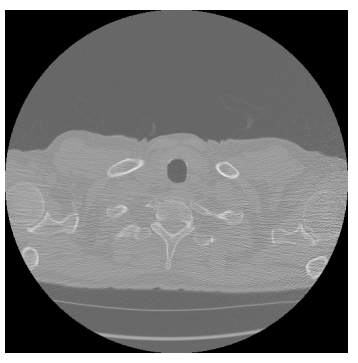

(a) $\mathrm{CT}$
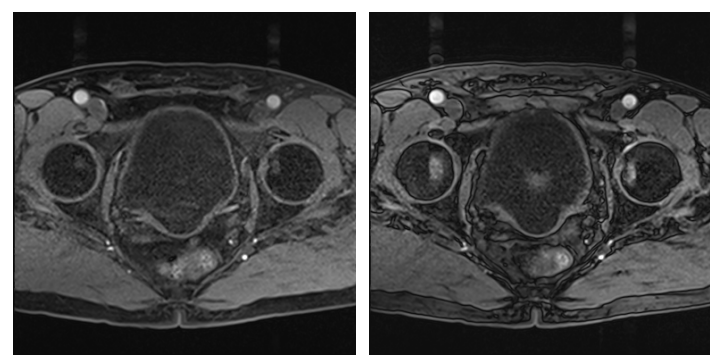

(b) MRI

Fig. 1: The challenge of the distinguishability for different VMIs: (a) Two whole-lung CT images from different patients are visually similar (b) Two prostate MRI images from different patients are also visually similar.

marking distinguishability and robustness. The contributions of our work can be highlighted as follows: 1) An improved zero-watermarking scheme is proposed to offer a lossless authenticity and copyright protection of VMI; 2) ring statistics are deployed to guarantee both the watermarking distinguishability and robustness; 3) an intra-slice variation based mechanism is designed to further enhance the watermarking distinguishability; 4) a logistic-logistic system based chaotic map is used to ensure the watermarking security and 5) we compare the proposed scheme with the state-of-the-art zero-watermarking schemes to demonstrate the superiority of our method.

The remainder of the paper is organized as follows. The related work is described in Section 2 to provide the literature review of our work. Section 3 describes the details of the proposed zero watermarking algorithm and how it enhances the watermarking distinguishability and robustness. In Section 4, the experimental results are presented to demonstrate the effectiveness of the proposed algorithm. Finally, Section 5 draws a conclusion and suggests the future work.

\section{The related work}

Digital watermarking is one of the most important technique for authenticity and copyright protection [41-43]. Three main categories of digital watermarking schemes are suitable for authenticity and copyright protection of medical images: 1) ROI lossless watermarking; 2) reversible watermarking; and 3) zero-watermarking. Each category is described individually as below.

ROI lossless watermarking schemes embed watermark into the region of noninterest (RONI) of medical images without distorting ROI to minimize the impact on general medical diagnosis. Memon et al. [10] propose a fragile watermarking technique, which embeds the watermark into the LSBs of RONI to protect the integrity of ROI. Nyeem et al. [11] define the edge pixels of medical images as RONI and embed the watermark into the LSBs of RONI to deal with ROI/RONI segmentation problem. Thanki et al. [12] use image processing operations such as image subtraction, image addition, and image complement for watermark embedding to reduce the computational complexity. Mousavi et al. [13] propose a watermarking method 
that can withstand high density of salt and pepper noise by combining channel coding, spatial domain watermarking method and noise filtering scheme. Ur et al. [14] design a spatial domain based hybrid watermarking technique using product codes, chaos theory and residue number system (RNS) to improve the watermarking security. Sharifara et al. [15] utilize 2-dimensional discrete wavelet transform (2D DWT) to yield a reliable authentication of medical images. Maheshkar et al. [16] use IWTSVD hybrid transform for embedding multiple watermark into RONI to enhance the watermark robustness. Solanki et al. [17] design a reliable method which uses DWT approach for hiding the RSA encrypted watermark into RONI part of the medical image to improve the watermarking security. Swaraja et al. [18] propose a regionbased firefly optimized algorithm that integrates DWT and Schur transformation for watermark embedding to further improve the watermark robustness.

Reversible watermarking schemes ensure the lossless recovery of the original medical imaging after watermarking extraction. Golpîra et al. [19] propose a scalable and reversible blind watermarking method for medical images, which uses histogram shifting in the wavelet domain to increase the capacity and image quality. Parah et al. [20] use image interpolation and embed watermarks into non-seeded pixels to improve the reversibility. Qasim et al. [21] utilize the difference expansion (DE) technique for embedding the watermark data into the ROI to detect intentional and unintentional changes in images. Arsalan et al. [22] embed the watermark into the LSBs of IWT coefficients and use genetic programming (GP) for intelligent wavelet coefficient selection to make an trade-off between imperceptibility and capacity. Priya et al. [23] propose a reversible watermarking method based on integer wavelet transform (IWT) and discrete gould transform (DGT) to improve the robustness. Turuk et al. [24] design a reversible method based on hybrid texture-quantization for embedding multiple watermark to enhance the confidentiality and reliability. Balasamy et al. [25] propose a medical image authentication scheme based on particle swarm optimization (PSO) and wavelet decomposition to achieve an balance between imperceptibility and embedding capacity. Lei et al. [26] utilize recursive dither modulation (RDM) algorithm after singular value decomposition (SVD) and wavelet transform to embed watermark. In addition, differential evolution (DE) is used to optimize the balance between imperceptibility, capacity, and robustness. Thabit et al. [27] modify the difference between the average value of high-frequency subbands and the average value of low-frequency subbands in the SLT domain to obtain improved invisibility, robustness, and capacity. Ansari et al. [28] design a watermarking scheme, which uses artificial bee colony controlling the embedding strength to obtain better imperceptibility. Liu et al. [29] utilize recursive dither modulation (RDM) combined with singular value decomposition and Slantlet transform to achieve better robustness.

Zero-watermarking schemes generate relationships between the medical image features and watermarks instead of embedding the watermark into the medical image directly, which ensures the medical image is distortion free. Singh et al. [30] propose a zero-watermarking scheme which utilizes singular value coefficients after wavelet transform to generate watermark. Han et al. [31, 32] design a robust zerowatermarking system for medical volume data which combines three-dimensional discrete cosine transform (3D-DCT) and the chaotic neural network. They fur- 
ther extend these 3D-DCT based methods into three-dimensional dual tree complex wavelet transform (3D-DTCWT) domain [33] to enhance the watermarking robustness. Fan et al. [34] propose a medical image watermarking algorithm based on the significant difference of cellular automata transform (CAT) for copyright protection to achieve sufficient security and robustness. To improve the robustness against geometric attacks, watermarking based on geometric invariant feature is proposed. Vellaisamy et al. [35, 36] utilize the first three and six sign bits of Hu's invariant moments in the contourlet transform (CT)-SVD domain of medical images, respectively. Xia et al. $[37,38]$ propose two lossless watermarking system for copyright protection of color medical image, which uses quaternion polar harmonic transforms (QPHTs) and Precise Quaternion polar harmonic fourier moments (PQPHFM) respectively. To improve the feature distinguishability, Liu et al. [39] utilize the completed local binary pattern (CLBP) operator to extract the content-based features of medical images. Zou et al. [40] design a zero-watermarking scheme by using the feature based on the gray scale variations in fan-ring partition.

Zero-watermarking outperforms the ROI lossless watermarking and reversible watermarking in terms of image quality and watermark robustness and thus is the most important and promising scheme for protecting medical images. However, none of the current state-of-the-art zero-watermarking methods can ensure both sufficient discriminative capability and robustness against geometrical attacks, such as flipping and rotations, simultaneously. In contrary, our proposed scheme not only satisfies the lossless quality requirement but also enhances both the watermarking distinguishability and robustness, especially against geometrical attack, which is one of the most typical attacks in copyright protection applications.

\section{The proposed scheme}

Our proposed scheme includes two phases: a registration phase and an identification phase. In the registration phase, the authenticity and copyright protection information of VMI will be registered in the certificate authority (CA) database. In the identification phase, the authenticity and copyright of the queried VMI will be verified. The details of each phase are described below.

\subsection{The registration phase}

The flowchart for the registration phase is shown in Fig. 2. In this phase, a scrambled multi-slice feature of VMI is first extracted. Then, master shares and ownership shares are generated. Finally, the ownership shares and secret keys for feature scrambling are stored for copyright identification.

\subsubsection{Feature extraction}

In our study, ring statistics are deployed to guarantee both the watermarking distinguishability and robustness. In addition, an intra-slice variation based mecha-

nism is designed to further enhance the watermarking distinguishability. Finally, a logistic-logistic system based chaotic map is used to ensure the watermarking security. The detailed feature extraction steps are given below. 


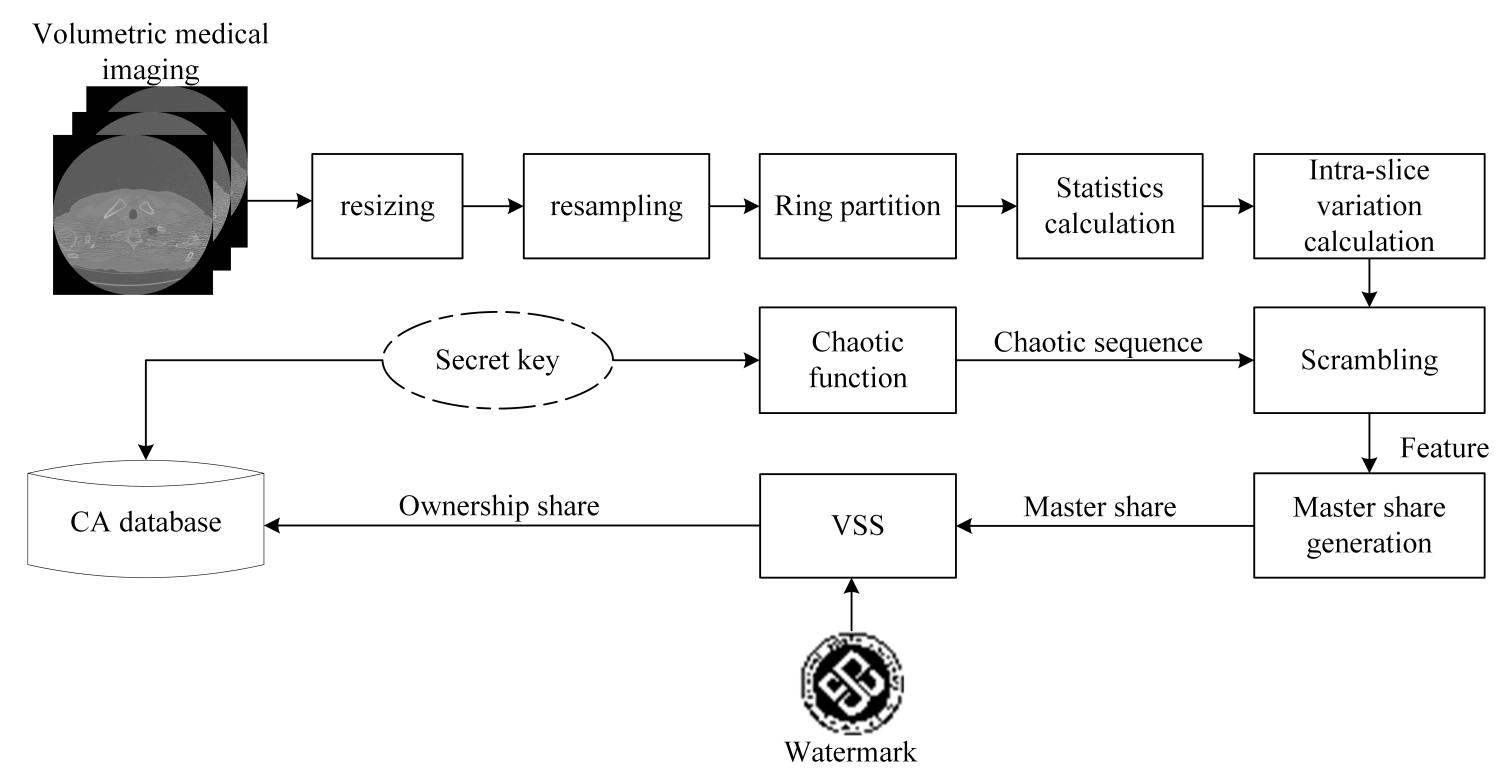

Fig. 2: The registration phase of our proposed scheme

Step 1: Normalize the slice size of VMI to $512 \times 512$ pixels to ensure the robustness against the resizing attacks.

Step 2: Resample the VMI to $L$ slices $I_{l}$, where $l=1,2, \ldots, L$.

These above 2 steps are the preprocessing of feature extraction. For each slice of $I_{l}$, we then perform steps 3-8 to get an one-slice feature.

Step 3: Take the center position of the slice as the center point, a circular area with a radius $R$ is selected as the target area to extract feature as shown in Fig. 3(c).

Step 4: Divide the target area into a central circle with radius $r$ and $N-1$ concentric rings with width $r$, where $N=\lfloor R / r\rfloor$. For each pixel $(i, j)$ in the target area, its partition $k$ is calculated as shown in (1).

$$
k=\left\lceil\frac{\sqrt{\left(i-i_{o}\right)^{2}+\left(j-j_{o}\right)^{2}}}{r}\right\rceil
$$

where $\left(i_{o}, j_{o}\right)$ is the center point of this target area, $1 \leq k \leq N$.

In this manner, the pixels still remain in their original partitions when the slice is rotated or flipped. This characteristic guarantees the sufficient robustness against rotation and flipping. Note that the circles in texture-rich central regions are designed to have smaller size comparing to those peripheral circles so that these circles are more salient to extract statistical features. Therefore, this design could further improve the distinguishability of the proposed scheme. In addition, we only use the pixels within the target area. The regions outside the largest ring, as shown in Fig. 3(c), are not used in our scheme for the following two reasons. (i) These regions are the most common places for the cropping attacks. Therefore, they are discarded to enhance the robustness against the cropping attacks. And (ii) the remained regions are more texture-rich, which are salient enough to keep the distinguishability.

Step 5: Calculate the four statistics: mean $\mu$, variance $\delta$, skewness $s$, and kurtosis $w$ of each partition based on (2)-(5). The mean $\mu_{k}$ indicates average energy of the $k_{t h}$ 


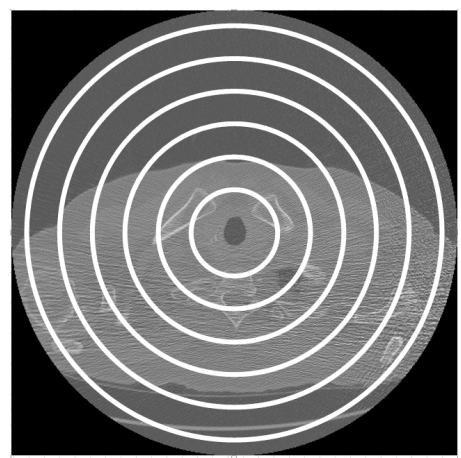

(a) $\mathrm{CT}$

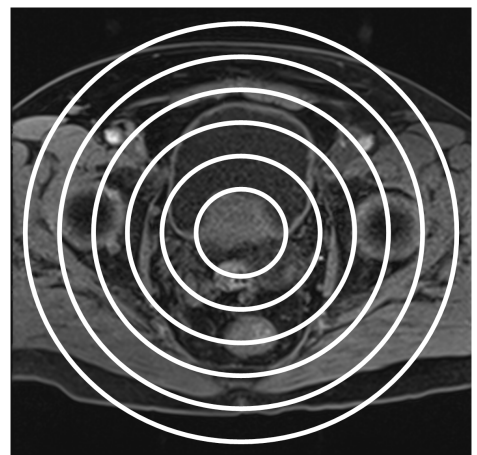

(b) MRI

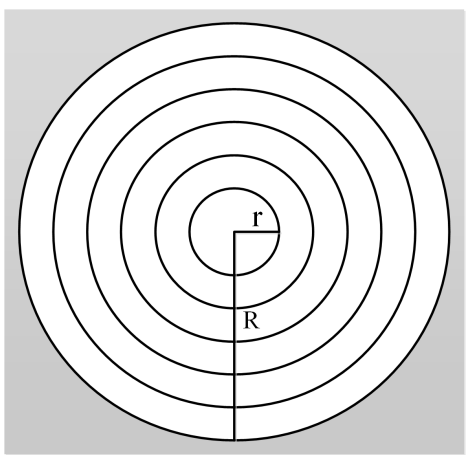

(c) Mask

Fig. 3: Examples of ring partition: A circle with radius $\mathrm{r}$ and $\mathrm{N}-1$ rings with width $\mathrm{r}$

partition, the variance $\delta_{k}$ reflects pixel fluctuation of the $k_{t h}$ partition, the skewness $s_{k}$ measures asymmetry of pixel values around the mean, and the kurtosis $w_{k}$ indicates the shape of the distribution of the $k_{t h}$ partition.

$$
\begin{gathered}
\mu_{k}=\frac{1}{N_{k}} \sum_{i=1}^{N_{k}} P_{k}(i) \\
\delta_{k}=\frac{1}{N_{k}-1} \sum_{i=1}^{N_{k}}\left(P_{k}(i)-\mu_{k}\right)^{2} \\
s_{k}=\frac{\frac{1}{N_{k}} \sum_{i=1}^{N_{k}}\left(P_{k}(i)-\mu_{k}\right)^{3}}{\sqrt{\left.\frac{1}{N_{k}} \sum_{i=1}^{N_{k}}\left(P_{k}(i)-\mu_{k}\right)^{2}\right)^{3}}} \\
w_{k}=\frac{\frac{1}{N_{k}} \sum_{i=1}^{N_{k}}\left(P_{k}(i)-\mu_{k}\right)^{4}}{\left(\frac{1}{N_{k}} \sum_{i=1}^{N_{k}}\left(P_{k}(i)-\mu_{k}\right)^{2}\right)^{2}}
\end{gathered}
$$

where $P_{k}$ is the $k_{t h}$ partition of image, $N_{k}$ is the total number of pixels in $P_{k}$, and $P_{k}(i)$ is the $i_{t h}$ pixel of $P_{k}, k=1,2, \ldots, N$. The use of these four statistical low-order moments ensures a remarkable trade-off between robustness and distinguishability. In particular, the mean value is to enhance the robustness, and the other three (variance, skewness and kurtosis) are to enhance the distinguishability.

Step 6: Calculate the residuals of statistics between the adjacent partitions based on (6)-(9) to reflect the intra-slice variations.

$$
D_{\mu}(k)=\mu_{k+1}-\mu_{k}
$$




$$
\begin{gathered}
D_{\delta}(k)=\delta_{k+1}-\delta_{k} \\
D_{s}(k)=s_{k+1}-s_{k} \\
D_{w}(k)=w_{k+1}-w_{k}
\end{gathered}
$$

where $k=1,2, \ldots, N-1$. Consider that the VMI images from same modality share similar structures which may lead to unacceptable distingushability. By calculating the intra-slice variations using (6)-(9), the detailed differences can be exaggerated to enhance the distinguishability across individual images from same modality.

Step 7: Binarize the residuals of statistics based on their own median values as shown in (10)-(13).

$$
\begin{aligned}
& B_{\mu}(k)= \begin{cases}1, & \text { if } D_{\mu}(k)>T_{\mu} \\
0, & \text { otherwise }\end{cases} \\
& B_{\delta}(k)= \begin{cases}1, & \text { if } D_{\delta}(k)>T_{\delta} ; \\
0, & \text { otherwise }\end{cases} \\
& B_{s}(k)= \begin{cases}1, & \text { if } D_{s}(k)>T_{s} ; \\
0, & \text { otherwise }\end{cases} \\
& B_{w}(k)= \begin{cases}1, & \text { if } D_{w}(k)>T_{w} ; \\
0, & \text { otherwise }\end{cases}
\end{aligned}
$$

where $T_{\mu}, T_{\delta}, T_{s}$ and $T_{w}$ are the median values of $\mu, \delta, s$ and $w$.

Step 8: Concatenate $B_{\mu}(k), B_{\delta}(k), B_{s}(k), B_{w}(k)$ as vector $f_{l}$ to represent the feature of one slice of $I_{l}$. Here, $k=1,2, \ldots, N-1$.

Step 9: Generate a multi-slice feature vector $V$ based on (14). The dimension of the feature vector $V$ is $4 \times(N-1) \times L$ bits.

$$
V=\left[f_{1}\left\|f_{2}\right\| \ldots \| f_{l}\right]
$$

where $\|$ represents the concatenation of features.

Step 10: Generate a chaotic sequence $H=\left\{h_{i+t}, 1 \leq i \leq 4 \times(N-1) \times L\right\}$ using logistic-logistic system (LLS) to ensure watermarking security. This system is one of the hybrid systems defined in [44] by combining two chaotic maps as shown in (15). In our study, we combine two logistic maps defined in (16) and strictly follow the parameter setting in [44] to ensure the effectiveness of chaotic system. According to [44], the hybrid system has better chaotic performance, and thus achieves better security than using single chaotic map. 


$$
h_{n+1}=E\left(u, h_{n}, v\right)=E_{\text {chaos }}\left(u, h_{n}\right) \times T(v)-\text { floor }\left(E_{\text {chaos }}\left(u, h_{n}\right) \times T(v)\right)
$$

with $T(v)=2^{v}$, and $E_{\text {chaos }}\left(u, h_{n}\right)$ is the logistic map:

$$
E_{\text {chaos }}\left(u, h_{n}\right)=h_{n+1}=u \times h_{n} \times\left(1-h_{n}\right)
$$

where $u, v$ are two control parameters, $0 \leq u \leq 10,8 \leq v \leq 20, h_{0}$ is the initial value of chaotic map, $n$ is the iteration number and $h_{n}$ is the output chaotic sequence. $H$ is a subsequence of length $4 \times(N-1) \times L$ in $h_{n} . u, v, h_{0}$ and $t$ are used together as the secret key. Here, $t$ is set to 500 because the chaotic sequences generated after multiple iterations have better chaotic performance.

Step 11: Binarize the chaotic sequence $H$ to obtain a binary chaotic sequence $B H=\left\{b h_{i}, 1 \leq i \leq 4 \times(N-1) \times L\right\}$ as follows.

$$
b h_{i}= \begin{cases}1, & \text { if } h_{i+t}>T \\ 0, & \text { otherwise }\end{cases}
$$

where $T$ is the average value of $H$.

Step 12: Generate a scrambled multi-slice feature $F$ by applying an XOR operation $(\oplus)$ between the binary chaotic sequence $B H$ and the multi-slice feature vector $V$.

$$
F=V \oplus B H
$$

In our study, both the feature distinguishability and robustness are guaranteed by using the ring statistics and intra-slice variations. To further prove this point, we illustrate some examples in Fig. 4 and Fig. 5. On one hand, we calculate the hamming distances between features of four VMIs (four CT and four MRI images respectively) from different patients as shown in Fig. 4(a) and Fig. 5(a). On the other hand, we calculate the distances between features of the four original VMIs and the four VMIs after attacks (resize 50\%, crop 15\%, rotation $90^{\circ}$ and horizontal flip) as shown in Fig. 4(b) and Fig. 5(b). Larger off-diagonal distances (gray colored) in Fig. 4(a) and Fig. 5(a) indicate better distinguishability while smaller diagonal distances (blue colored) in Fig. 4(b) and Fig. 5(b) demonstrate stronger robustness. As shown in Fig. 4(a) and Fig. 5(a), although the four VMIs are visually similar, the hamming distance values of our designed feature are still very large, thus confirming the sufficient distinguishability of our designed feature. As shown in Fig. 4(b) and Fig. 5(b), the value on the diagonal is close to 0 and much smaller than the value at other positions, thus confirming the strong robustness of our designed feature.

\subsubsection{Generation of Master and Ownership Shares}

In our study, we deploy $(2,2)$ visual secret sharing (VSS) [45] to generate master and ownership shares. As shown in Fig. 6, each pixel of the binary watermark image is transformed into two $2 \times 2$ blocks in $(2,2)$ VSS. The two blocks from a white pixel 


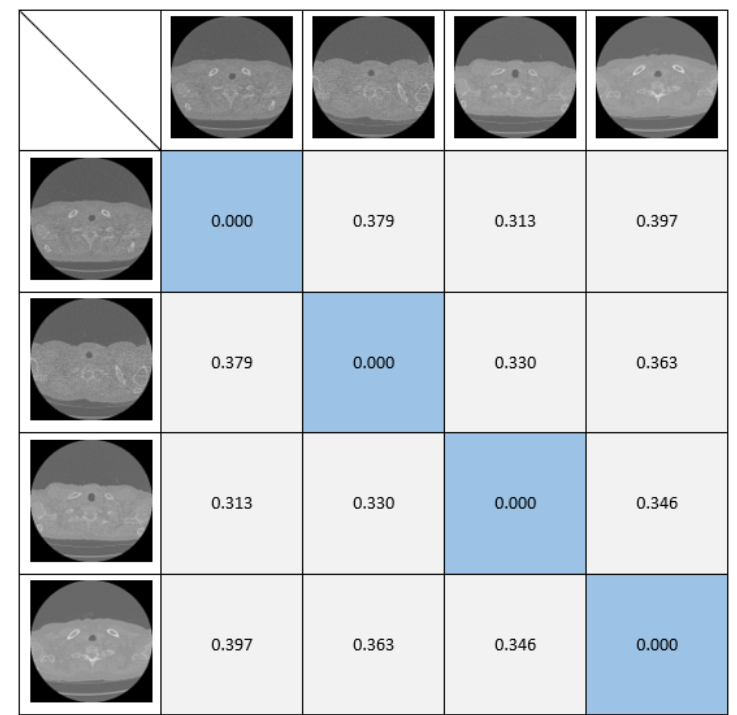

(a)

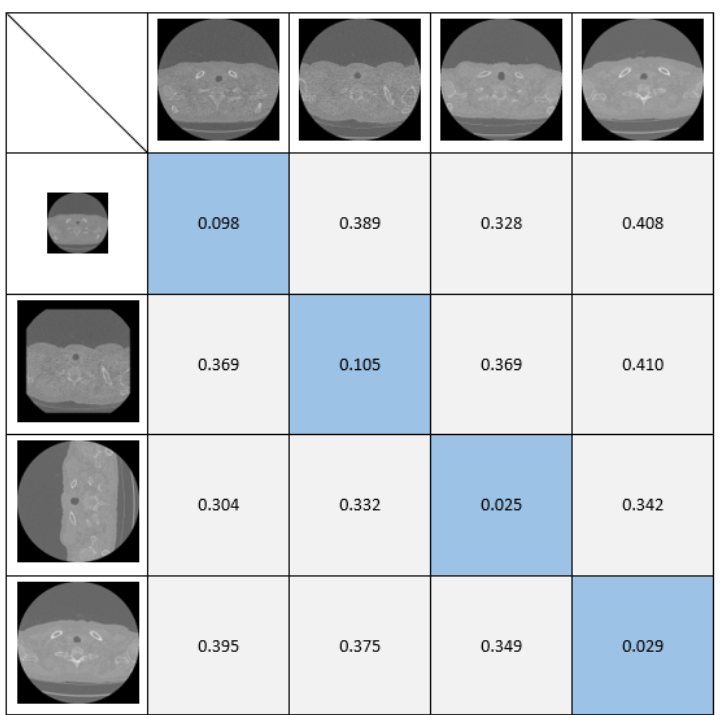

(b)

Fig. 4: Examples to demonstrate the distinguishability and robustness of our designed feature on CT images

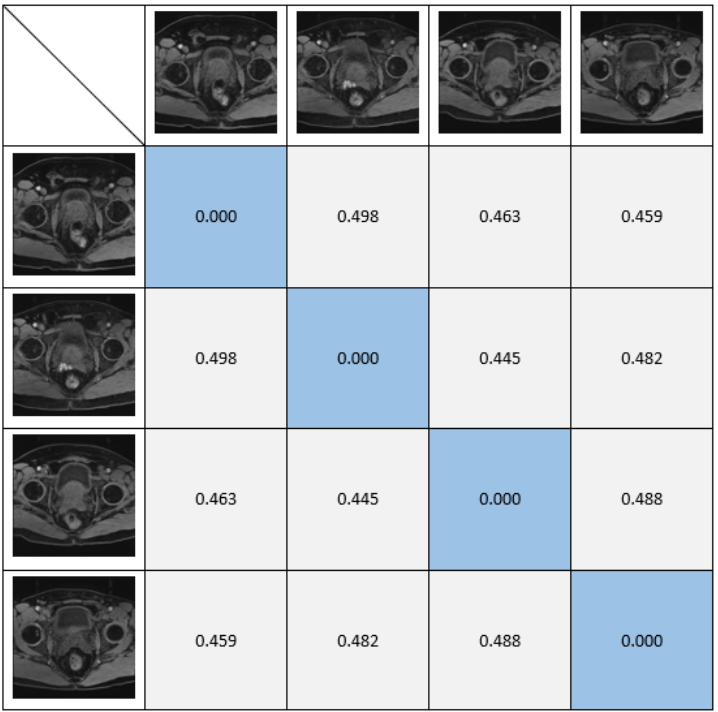

(a)

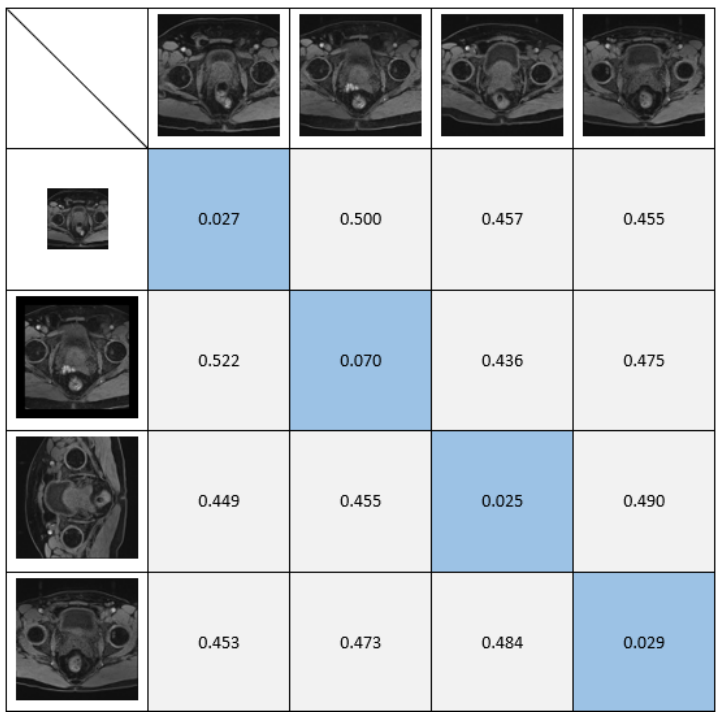

(b)

Fig. 5: Examples to demonstrate the distinguishability and robustness of our designed feature on MRI images

are the same, whereas those from a black pixel are complementary. The detailed share generation steps are given below.

Step 1: Rearrange the feature vector $F$ into a binary matrix $G$ with size of $m \times m$.

Step 2: Generate master share $M$ with size of $2 m \times 2 m$ by converting each element 


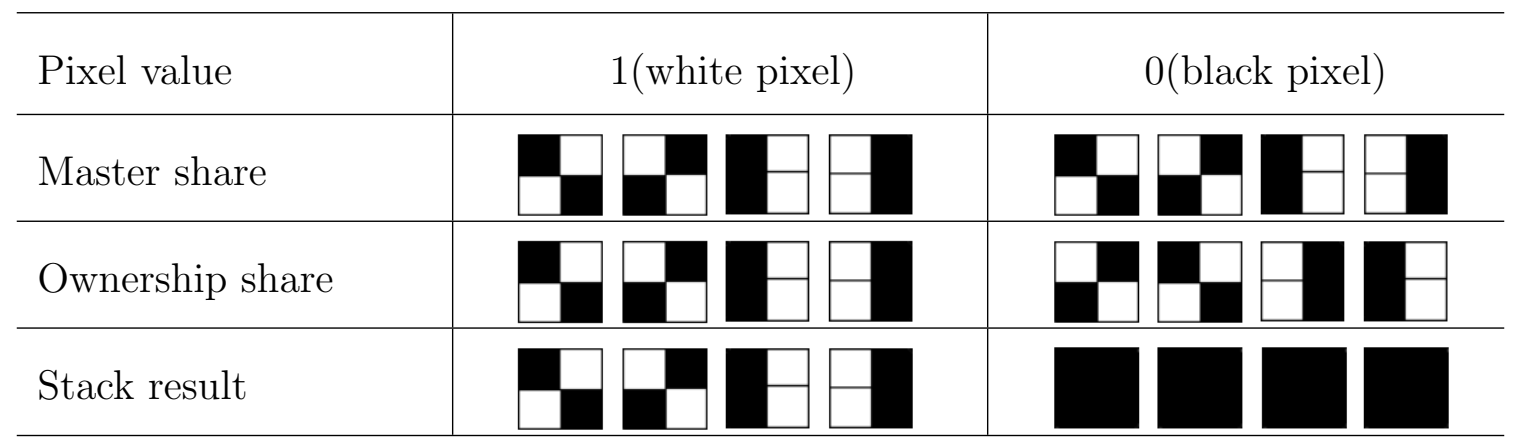

Fig. 6: The $(2,2)$ VSS method

$G(i, j)$ in matrix $G$ into a $2 \times 2$ matrix. The conversion rules are shown in (19).

$$
M(i, j)= \begin{cases}{\left[\begin{array}{ll}
1 & 0 \\
0 & 1
\end{array}\right],} & G(i, j)=1 \\
{\left[\begin{array}{ll}
0 & 1 \\
1 & 0
\end{array}\right],} & G(i, j)=0\end{cases}
$$

Step 3: Generate the ownership share $O$ with size of $2 m \times 2 m$ by using the $(2,2)$ VSS method as shown in (20).

$$
O(i, j)= \begin{cases}{\left[\begin{array}{ll}
0 & 1 \\
1 & 0
\end{array}\right],} & W(i, j)=1, M(i, j)=\left[\begin{array}{ll}
0 & 1 \\
1 & 0
\end{array}\right] ; \\
{\left[\begin{array}{ll}
1 & 0 \\
0 & 1
\end{array}\right],} & W(i, j)=1, M(i, j)=\left[\begin{array}{ll}
1 & 0 \\
0 & 1
\end{array}\right] ; \\
{\left[\begin{array}{ll}
0 & 1 \\
1 & 0
\end{array}\right],} & W(i, j)=0, M(i, j)=\left[\begin{array}{ll}
1 & 0 \\
0 & 1
\end{array}\right] ; \\
{\left[\begin{array}{ll}
1 & 0 \\
0 & 1
\end{array}\right],} & W(i, j)=0, M(i, j)=\left[\begin{array}{ll}
0 & 1 \\
1 & 0
\end{array}\right] ;\end{cases}
$$

where $1 \leq i \leq m, 1 \leq j \leq m$ and $W(i, j)$ represents the pixel of the $m \times m$ watermark image.

Step 4: Store the ownership share $O$ together with its secret key into the CA database to provide the basis for authenticity and copyright identification.

\subsection{The identification phase}

In this phase, the watermark is first recovered based on the queried VMI and its corresponding ownership share. Then, the authenticity and copyright of queried VMI is verified by comparing the original watermark with the recovered watermark. 


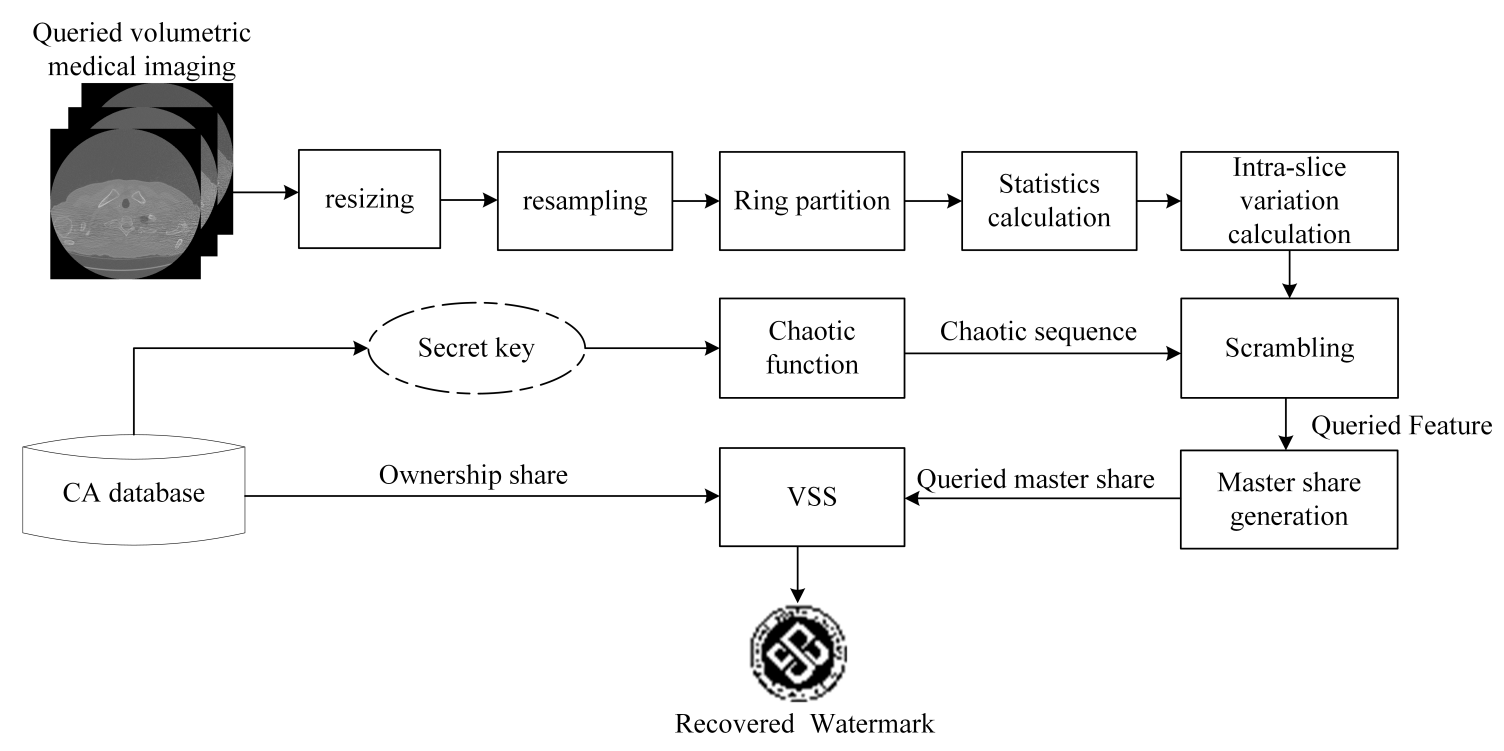

Fig. 7: The identification phase of our proposed scheme

The flowchart for the identification phase is shown in Fig. 7 and the detailed steps are given below.

Step 1: Extract the multi-slice feature vector $V^{\prime}$ of the queried VMI following the steps 1-9 in section 3.1.1.

Step 2: Obtain the binary chaotic sequence $B H$ by using the stored key following the steps 10-11 in section 3.1.1.

Step 3: Generate a scrambled multi-slice feature $F^{\prime}$ of the queried VMI following the step 12 in section 3.1.1.

Step 4: Generate the queried master share $M^{\prime}$ following the steps 1-2 in section 3.1.2.

Step 5: Generate the intermediate matrix $S$ by stacking the queried master share $M^{\prime}$ and the corresponding ownership share $O$, following the $(2,2)$ VSS method.

Step 6: Recover the watermark $W^{\prime}$ from $S$ based on (21).

$$
W^{\prime}(i, j)= \begin{cases}1, & \text { if } \sum s_{i, j}(x, y) \geq 2 \\ 0, & \text { otherwise }\end{cases}
$$

where $s_{i, j}(x, y)$ are non-overlapping $2 \times 2$ blocks of $S, 1 \leq i, j \leq m$ and $1 \leq x, y \leq 2$.

Step 7: Identify the authenticity and copyright of the queried VMI by calculating the bit error rate (BER) between the original watermark $W$ and the recovered watermark $W^{\prime}$ as shown in (22).

$$
B E R=\frac{\sum W(i, j) \oplus W^{\prime}(i, j)}{m \times m}
$$

where $W(i, j)$ and $W^{\prime}(i, j)$ represent the pixels of the original and recovered watermarks, respectively. 


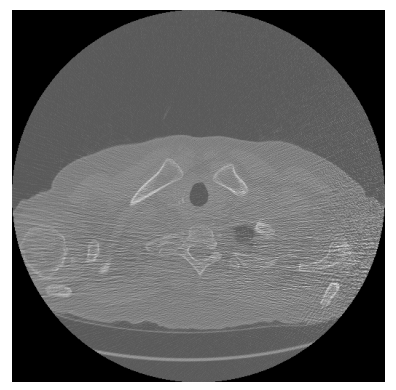

(a)

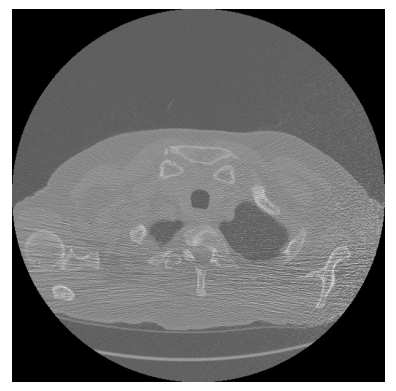

(d)

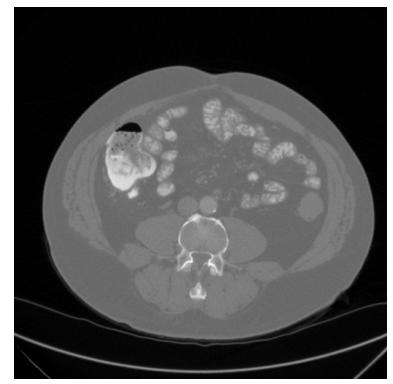

(b)

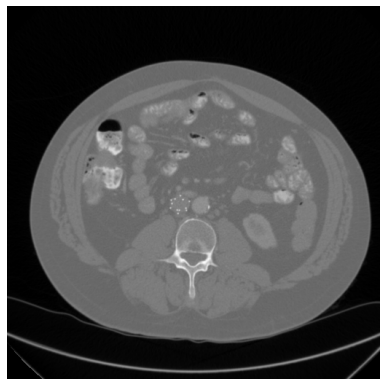

(e)

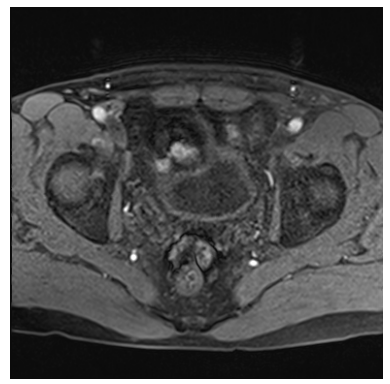

(c)

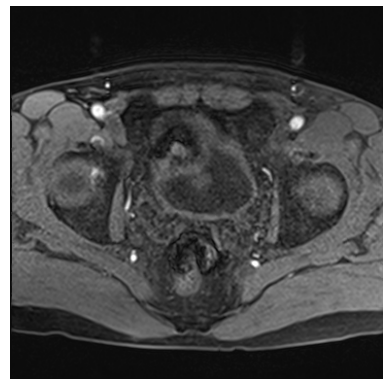

(f)

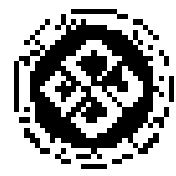

(g)

Fig. 8: Examples of the experimental images. (a) and (d) are two CT images from the ELCAP database, (b) and (e) are two CT images from the TCIA Public Access, (c) and (f) are two MRI images from the Nottingham hospital database, and $(\mathrm{g})$ is the watermark image.

\section{Experimental results}

\subsection{Experimental settings}

Our experimental data consists of 400 VMIs, including 200 Computed Tomography (CT) images and 200 Magnetic Resonance Images (MRI). CT images are from the Early Lung Cancer Action Program (ELCAP) database [46] and the Cancer Imaging Archive (TCIA) Public Access [47]. Images of the former database have $\sim 300$ slices with resolution $512 \times 512$ while images of the latter one are of size $512 \times 512$ pixels, each contains 500-700 slices. Magnetic Resonance Images (MRI) are collected by Nottingham university in the prostate cancer imaging interpretation training project [48] named Nottingham hospital database. These images have $\sim 25$ slices with resolution $512 \times 512$. The examples from three different database and the watermark image are illustrated in Fig. 8. We separate this two VMI modalities as two individual testing datasets in all our experiments for effective evaluations and fair comparisons. Both CT and MRI images suffer 27 attacks including compression, contrast and brightness changes, noise addition, smoothing and geometric transformations. In this manner, $5400 \mathrm{CT}$ images and $5400 \mathrm{MRI}$ images are generated as queried VMIs for our experiments. All attacks and their parameters are detailed in Table 1. In our experiments, the initial value $h_{0}$ and two control parameters $u, v$ of the deployed LLS are set to $0.456,5.4321$ and 14, respectively. This setting strictly follows [44] to ensure the effectiveness of the chaotic system. 
Table 1: Attack types and their parameters

\begin{tabular}{ll}
\hline Attack types & Parameters \\
\hline JPEG compression (JC) & Quality factor $=\{30 \%, 50 \%\}$ \\
\hline Contrast adjustment (CA) & $\{-20 \%,+20 \%\}$ \\
Brightness adjustment (BA) & $\{-20 \%,+20 \%\}$ \\
\hline Gaussian noise (GN) & Mean $=0$, variance $=\{0.01,0.02\}$ \\
Salt and pepper noise (SPN) & Noise density $=\{0.01,0.02\}$ \\
\hline Gaussian filtering (GF) & Variance $=1$, window $=\{3 \times 3,5 \times 5\}$ \\
Average filtering (AF) & Window $=\{3 \times 3,5 \times 5\}$ \\
Median filtering (MF) & Window $=\{3 \times 3,5 \times 5\}$ \\
\hline Resizing (RS) & $\{50 \%, 200 \%\}$ \\
Crop from image edges (CIE) & $\{15 \%, 20 \%\}$ \\
Shifting(SF) & $\{$ Horizontal: $1 \%$, Vertical: $1 \%\}$ \\
Rotation $(\mathrm{RT})$ & $\left\{45^{\circ}, 60^{\circ}, 90^{\circ}\right\}$ \\
Flipping (FL) & $\{$ Horizontal, Vertical $\}$ \\
\hline
\end{tabular}

\subsection{Parameter discussion}

In our scheme, parameters are selected according to the following criteria. Firstly, the radius of target area $R$ is selected to be less than 0.475 of the width of resized image to discard the regions outside the largest ring, which enhances the robustness against the cropping attacks. Secondly, the size of our extracted feature is equal to that of watermark as shown in (23) to ensure the effective mapping between our extracted feature and the deployed watermark. Thirdly, the watermark size $m \times m$ is selected to $32 \times 32$ by considering the balance between computation complexity and amount of information for authenticity and copyright identification, which is a usual setting in watermarking applications such as [37, 49-51].

$$
L \times(\lfloor R / r\rfloor-1) \times 4=L \times(N-1) \times 4=m \times m
$$

To evaluate watermarking performances with different parameters, we compare three groups of parameters as shown in Table 2.

Table 2: Groups of parameters

\begin{tabular}{lcccc}
\hline Description & Abbreviation & Group1 & Group2 & Group3 \\
\hline Number of downsampled slices & $L$ & 8 & 32 & 16 \\
Radius of target area & $R$ & 231 & 234 & 238 \\
Width of concentric rings & $r$ & 7 & 26 & 14 \\
Number of ring partitions & $N$ & 33 & 9 & 17 \\
\hline
\end{tabular}


We first use inter-BERs to evaluate the watermarking distinguishability. InterBERs is defined as the BER between the genuine and fake watermarks. Here, the genuine watermark is generated by stacking the master share and the ownership share of the same VMI while the fake watermark is generated by stacking the shares of different VMIs. In our experiment, inter-BERs are calculated from the 200 different VMIs of a same modality. As a result, there are totally $200 \times 199=39800$ inter-BERs with 39800/2=19900 different values for CT (MRI) images. A larger inter-BER value indicates better watermarking distinguishability. In addition, we use the intraBERs to evaluate the watermarking robustness. The intra-BER is calculated as the BER between the original watermark and the recovered watermarks under various attacks. A smaller intra-BER indicates better watermarking robustness. Finally, we calculate the false positive rate $P_{f p}$ and the false negative rate $P_{f n}$ to evaluate the overall watermarking performance. The $P_{f p}$ is defined as the probability of identifying two different CT (MRI) images as a same CT (MRI) image. It indicates the distinguishability of our proposed scheme. The $P_{f n}$ is defined as the probability of identifying the original CT (MRI) image and its attacked format as two different VMIs. It manifests the robustness of our proposed scheme. The $P_{f p}$ and $P_{f n}$ are defined as (24) and (25) respectively.

$$
\begin{gathered}
P_{f p}=\frac{N_{f p}}{N_{t d}} \\
P_{f n}=\frac{N_{f n}}{N_{t s}}
\end{gathered}
$$

Here, $N_{f p}$ is the number of different CT (MRI) images whose inter-BER is smaller than a predefined threshold $T, N_{t d}$ represents the true number of different pairs of CT (MRI) images, $N_{f n}$ is the number of pairs of CT (MRI) image and its attacked format whose intra-BER is larger than $T$, and $N_{t s}$ is the true number of attacked CT (MRI) images. The threshold $T$ is determined by either $P_{f p}$ or $P_{f n}$. In our study, the threshold $T$ is determined by $P_{f p}$. The $P_{f p}$ is set to $1 / 19900$ (the smallest $P_{f p}$ except 0 in our experiment) for both CT and MR images to ensure the high distinguishability, and $P_{f n}$ is calculated to evaluate the overall performance under various attacks. A smaller $P_{f n}$ value indicates better overall watermarking performance. The results including the average value of inter-BERs of 39800 pairs of CT (MRI) images, average value of mean intra-BERs and average value of $P_{f n}$ of 5400 attacked CT (MRI) images under 27 attacks by using our proposed scheme of different parameters are given in Table 3.

From the last column (average $P_{f n}$ with $P_{f p}=1 / 19900$ ) in Table 3, it is obvious that the parameter setting of Group 3 achieves better trade-off between watermarking distinguishability and robustness (optical overall performance) than those of Group 1 (v.s. robustness) and Group 2 (v.s. distinguishability). Therefore, in the latter experiments, the parameters of Group 3 are set in our proposed scheme.

\subsection{Ablation study}

In this section, the effectiveness of ring-statistics calculation (RSC) and intra-slice variations (IASV), which are two of the key components of our scheme, are evaluated 
Table 3: Results with different parameters

\begin{tabular}{ccccc}
\hline & $\begin{array}{c}\text { Parameter } \\
\text { setting }\end{array}$ & $\begin{array}{c}\text { Average value of } \\
\text { inter-BER }\end{array}$ & $\begin{array}{c}\text { Average value of } \\
\text { mean intra-BER }\end{array}$ & $\begin{array}{c}\text { Average value of } \\
P_{f n}\left(P_{f p}=1 / 19900\right)\end{array}$ \\
\hline \multirow{3}{*}{ CT images } & Group1 & 0.4570 & 0.0687 & 0.0311 \\
& Group2 & 0.4290 & 0.0415 & 0.0148 \\
& Group3 & 0.4428 & 0.0515 & 0.0006 \\
\hline \multirow{3}{*}{ MRI images } & Group1 & 0.4942 & 0.0674 & 0.0196 \\
& Group2 & 0.4839 & 0.0397 & 0.0026 \\
& Group3 & 0.4870 & 0.0495 & 0.0017 \\
\hline
\end{tabular}

Table 4: Comparison of the inter-BERs with S1 and S2

\begin{tabular}{llcc}
\hline & & Average & Minimum \\
\hline \multirow{3}{*}{ CT images } & S1 (IASV+Fan-ring) & 0.3824 & 0.2007 \\
& S2 (RSC) & 0.3264 & 0.0762 \\
& Proposed Scheme (RSC+IASV) & 0.4428 & 0.2207 \\
\hline \multirow{3}{*}{ MRI images } & S1 (IASV+Fan-ring) & 0.4187 & 0.1743 \\
& S2 (RSC) & 0.4453 & 0.1309 \\
& Proposed Scheme (RSC+IASV) & 0.4870 & 0.1992 \\
\hline
\end{tabular}

by comparing our proposed scheme (RSC+IASV) with two other schemes, which are S1 (IASV+Fan-ring) and S2 (RSC). Here, both S1 and S2 use multi-slice feature. The S1 deploys the variations between average values of fan-ring partitions as in [40] instead of using RSC for feature extraction, while the S2 deploys ring-statistics without IASV for feature extraction.

\subsubsection{Evaluation of distinguishability}

We first use inter-BERs to evaluate the watermarking distinguishability. The inter-BERs of S1, S2 and our proposed scheme are listed in Table 4.

As we can see from Table 4, the average and minimum values of inter-BERs by using our proposed scheme are larger than the values of S1 and S2. These results demonstrate that the combination of RSC and IASV greatly improves the distinguishability.

\subsubsection{Evaluation of robustness}

Then, we use the intra-BERs to evaluate the watermarking robustness. The intra-BER is calculated as the BER between the original watermark and the recovered watermarks under various attacks. A smaller intra-BER indicates better watermarking robustness. The mean intra-BERs of S1, S2 and our proposed scheme are listed in Table 5 and Table 6.

As shown in Table 5 and Table 6, the average value of mean intra-BERs of our proposed scheme is much smaller than that of S1. In particular, for the rotation and 
Table 5: Comparison of the mean intra-BERs between the original watermark and the extracted watermarks with S1 and S2 on CT images

\begin{tabular}{|c|c|c|c|}
\hline Attack & $\begin{array}{c}\mathrm{S} 1 \\
(\mathrm{IASV}+\mathrm{Fan}-\text { ring })\end{array}$ & $\begin{array}{c}\mathrm{S} 2 \\
(\mathrm{RSC})\end{array}$ & $\begin{array}{l}\text { Proposed Scheme } \\
\quad(\mathrm{RSC}+\mathrm{IASV})\end{array}$ \\
\hline JC $30 \%$ & 0.0954 & 0.0161 & 0.0284 \\
\hline JC $50 \%$ & 0.0746 & 0.0108 & 0.0200 \\
\hline $\mathrm{CA}+20 \%$ & 0.0391 & 0.0296 & 0.0304 \\
\hline CA $-20 \%$ & 0.0121 & 0.0022 & 0.0024 \\
\hline $\mathrm{BA}+20 \%$ & 0.0069 & 0.0138 & 0.0224 \\
\hline $\mathrm{BA}-20 \%$ & 0.0118 & 0.0024 & 0.0028 \\
\hline GN 0.01 & 0.1551 & 0.0581 & 0.0940 \\
\hline GN 0.02 & 0.1797 & 0.0875 & 0.1303 \\
\hline SPN 0.01 & 0.0992 & 0.0683 & 0.0892 \\
\hline SPN 0.02 & 0.1254 & 0.0831 & 0.1092 \\
\hline $\mathrm{GF} 3 \times 3$ & 0.0329 & 0.0303 & 0.0382 \\
\hline GF $5 \times 5$ & 0.0392 & 0.0351 & 0.0460 \\
\hline $\mathrm{AF} 3 \times 3$ & 0.0387 & 0.0320 & 0.0411 \\
\hline $\mathrm{AF} 5 \times 5$ & 0.0598 & 0.0448 & 0.0638 \\
\hline $\mathrm{MF} 3 \times 3$ & 0.0503 & 0.0275 & 0.0354 \\
\hline $\mathrm{MF} 5 \times 5$ & 0.0687 & 0.0434 & 0.0626 \\
\hline RS $50 \%$ & 0.0407 & 0.0292 & 0.0371 \\
\hline RS $200 \%$ & 0.0136 & 0.0147 & 0.0150 \\
\hline CIE $15 \%$ & 0.0026 & 0.0455 & 0.0583 \\
\hline CIE $20 \%$ & 0.0123 & 0.0638 & 0.0748 \\
\hline $\mathrm{SF}(\mathrm{H}) 1 \%$ & 0.1817 & 0.0462 & 0.1099 \\
\hline $\mathrm{SF}(\mathrm{V}) 1 \%$ & 0.2051 & 0.0599 & 0.1385 \\
\hline $\mathrm{RT} 45^{\circ}$ & 0.4239 & 0.0096 & 0.0239 \\
\hline $\mathrm{RT} 60^{\circ}$ & 0.4192 & 0.0093 & 0.0250 \\
\hline RT $90^{\circ}$ & 0.4143 & 0.0105 & 0.0282 \\
\hline $\mathrm{FL}(\mathrm{H})$ & 0.3313 & 0.0105 & 0.0282 \\
\hline $\mathrm{FL}(\mathrm{V})$ & 0.3952 & 0.0141 & 0.0353 \\
\hline Average: & 0.1307 & 0.0333 & 0.0515 \\
\hline
\end{tabular}

flipping attacks, the mean intra-BERs of our proposed scheme are around 0.0300, whereas the values of $\mathrm{S} 1$ are around 0.4000 . These results demonstrate that RSC leads to improved robustness, especially against geometric attacks. Although S2 yields slightly better robustness than our proposed scheme, it is at the price of significantly worse distinguishability (smaller inter-BERs) as shown in Table 4. These results demonstrate that IASV significantly enhances the distinguishability without losing much robustness, thus leading to a better overall watermarking performance. 
Table 6: Comparison of the mean intra-BERs between the original watermark and the extracted watermarks with S1 and S2 on MRI images

\begin{tabular}{|c|c|c|c|}
\hline Attack & $\begin{array}{c}\mathrm{S} 1 \\
(\mathrm{IASV}+\mathrm{Fan}-\text { ring })\end{array}$ & $\begin{array}{c}\mathrm{S} 2 \\
(\mathrm{RSC})\end{array}$ & $\begin{array}{l}\text { Proposed Scheme } \\
\quad(\mathrm{RSC}+\mathrm{IASV})\end{array}$ \\
\hline JC $30 \%$ & 0.0391 & 0.0113 & 0.0229 \\
\hline JC $50 \%$ & 0.0267 & 0.0079 & 0.0149 \\
\hline $\mathrm{CA}+20 \%$ & 0.0301 & 0.0476 & 0.0570 \\
\hline CA $-20 \%$ & 0.0077 & 0.0017 & 0.0028 \\
\hline $\mathrm{BA}+20 \%$ & 0.0053 & 0.0130 & 0.0199 \\
\hline $\mathrm{BA}-20 \%$ & 0.0034 & 0.0018 & 0.0028 \\
\hline GN 0.01 & 0.0829 & 0.0479 & 0.0683 \\
\hline GN 0.02 & 0.1071 & 0.0713 & 0.0975 \\
\hline SPN 0.01 & 0.0417 & 0.0862 & 0.0835 \\
\hline SPN 0.02 & 0.0618 & 0.1109 & 0.1018 \\
\hline $\mathrm{GF} 3 \times 3$ & 0.0214 & 0.0208 & 0.0334 \\
\hline GF $5 \times 5$ & 0.0304 & 0.0294 & 0.0464 \\
\hline $\mathrm{AF} 3 \times 3$ & 0.0261 & 0.0247 & 0.0389 \\
\hline $\mathrm{AF} 5 \times 5$ & 0.0592 & 0.0510 & 0.0773 \\
\hline $\mathrm{MF} 3 \times 3$ & 0.0256 & 0.0191 & 0.0295 \\
\hline $\mathrm{MF} 5 \times 5$ & 0.0570 & 0.0435 & 0.0654 \\
\hline RS $50 \%$ & 0.0237 & 0.0173 & 0.0284 \\
\hline RS $200 \%$ & 0.0025 & 0.0013 & 0.0027 \\
\hline CIE $15 \%$ & 0.0020 & 0.0373 & 0.0544 \\
\hline CIE $20 \%$ & 0.0149 & 0.0714 & 0.0923 \\
\hline $\mathrm{SF}(\mathrm{H}) 1 \%$ & 0.1851 & 0.0498 & 0.1098 \\
\hline $\mathrm{SF}(\mathrm{V}) 1 \%$ & 0.1866 & 0.0670 & 0.1326 \\
\hline $\mathrm{RT} 45^{\circ}$ & 0.4382 & 0.0112 & 0.0258 \\
\hline $\mathrm{RT} 60^{\circ}$ & 0.4268 & 0.0112 & 0.0277 \\
\hline RT $90^{\circ}$ & 0.4163 & 0.0132 & 0.0311 \\
\hline $\mathrm{FL}(\mathrm{H})$ & 0.3685 & 0.0131 & 0.0308 \\
\hline $\mathrm{FL}(\mathrm{V})$ & 0.4270 & 0.0182 & 0.0375 \\
\hline Average: & 0.1154 & 0.0333 & 0.0495 \\
\hline
\end{tabular}

\subsubsection{Evaluation of overall performance}

We also plot the histograms of inter-BERs and intra-BERs reflecting the trade-off between distinguishability and robustness to evaluate the overall watermarking performance. A smaller overlapped region between the distributions of inter-BERs and intra-BERs indicates a better trade-off between distinguishability and robustness. The histograms of inter-BERs and intra-BERs of S1, S2 and our proposed scheme are plotted in Fig. 9 and Fig. 10.

As shown in Fig. 9 and Fig. 10 (a)-(c), our proposed scheme has insignificant 


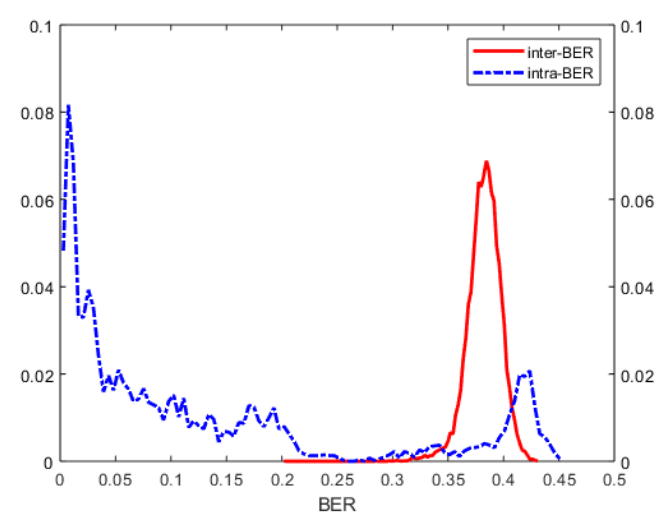

(a) Histograms of S1(IASV+Fan-ring)

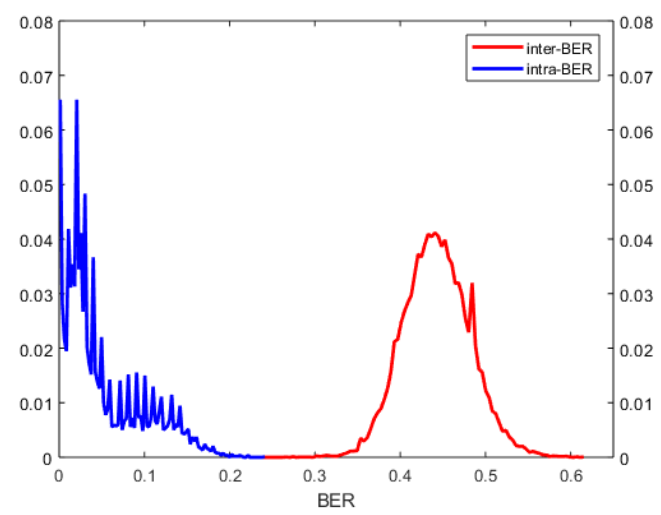

(c) Histograms of proposed Scheme(IASV+RSC)

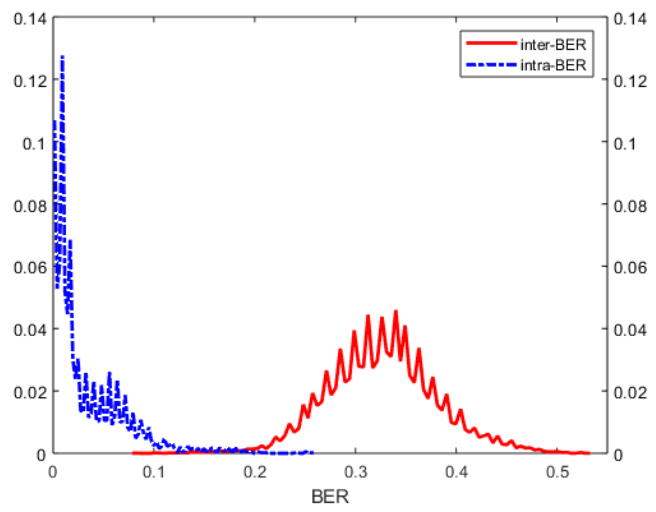

(b) Histograms of S2(RSC)

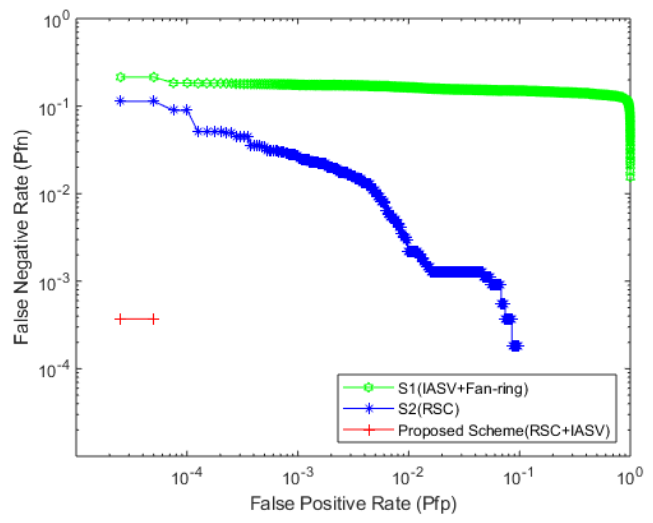

(d) DET graphs

Fig. 9: (a)-(c) are the histograms of inter-BER and intra-BER by using S1, S2 and our proposed scheme on CT images. A smaller overlapped region indicates a better trade-off between distinguishability and robustness. Our proposed scheme has insignificant overlapped region, whereas other schemes have different degrees of overlaps. (d) includes the DET graphs of S1, S2 and our proposed scheme on CT images. The curve closer to $(0,0)$ demonstrates the better overall watermarking performance. Note that any point of which $P_{f n}$ equals to 0 with different $P_{f p}$ (from $1 / 19900$ to 1 by increment of 1/19900) would not appear in the DET graphs. As shown in (d), the curve of our proposed scheme is continuously below those of other two schemes, confirming better performance on CT images compared to them.

overlapped region between the distribution of inter-BERs and intra-BERs, whereas other schemes have different degrees of overlaps. These results demonstrate that our proposed scheme yield the best trade-off between distinguishability and robustness among all these three schemes.

Furthermore, we calculate the false positive rate $P_{f p}$ and the false negative rate $P_{f n}$ to evaluate the overall watermarking performance. In our study, the $P_{f p}$ is set to 1/19900 (the smallest $P_{f p}$ except 0 in our experiment) for both CT and MRI images to ensure the high distinguishability, and $P_{f n}$ is calculated to evaluate the overall watermarking performance. The results of $P_{f n}$ calculated by using S1, S2 and our proposed scheme for both CT and MRI images are given in Table 7 and Table 8.

From Table 7 and Table 8 , we can see that the average value of $P_{f n}$ of our proposed 


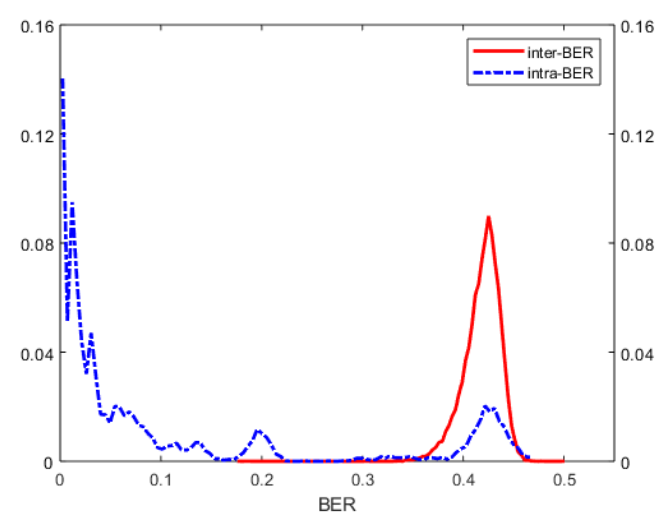

(a) Histograms of S1(IASV+Fan-ring)

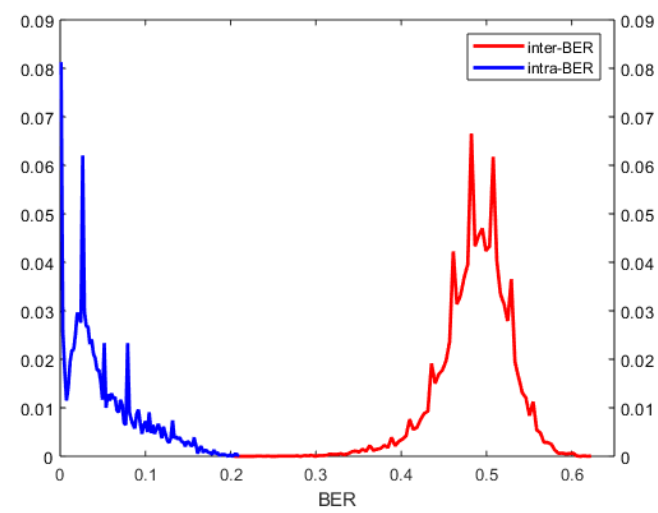

(c) Histograms of proposed Scheme(IASV+RSC)

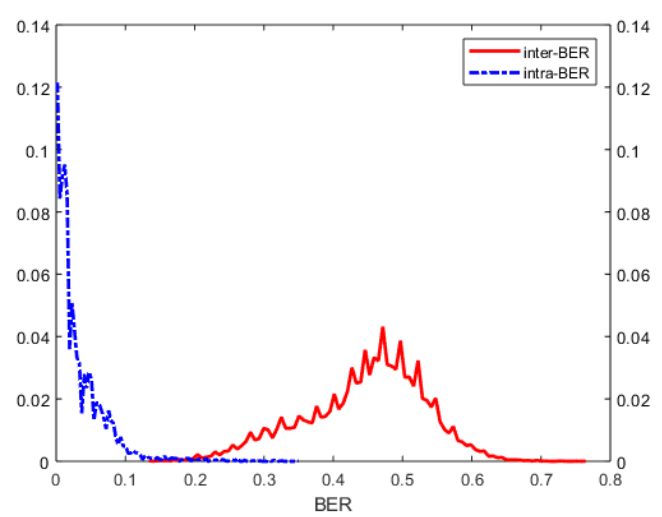

(b) Histograms of S2(RSC)

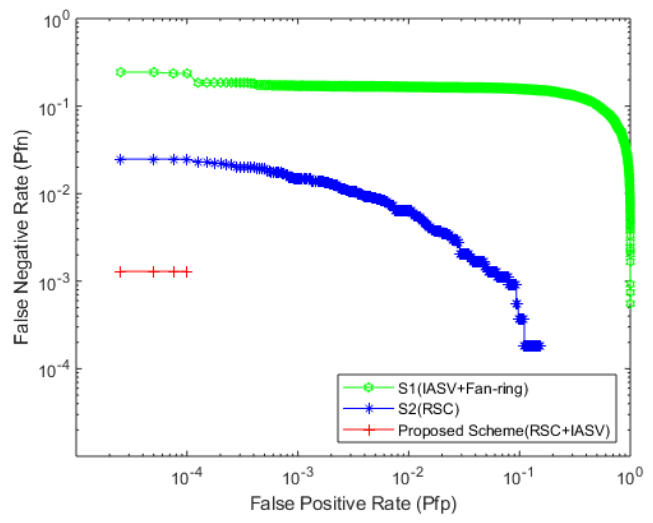

(d) DET graphs

Fig. 10: (a)-(c) are the histograms of inter-BER and intra-BER by using S1, S2 and our proposed scheme on MRI images. A smaller overlapped region indicates a better trade-off between distinguishability and robustness. Our proposed scheme has insignificant overlapped region, whereas other schemes have different degrees of overlaps. (d) includes the DET graphs of S1, S2 and our proposed scheme on MRI images. The curve closer to $(0,0)$ demonstrates the better overall watermarking performance. Note that any point of which $P_{f n}$ equals to 0 with different $P_{f p}$ (from $1 / 19900$ to 1 by increment of 1/19900) would not appear in the DET graphs. As shown in (d), the curve of our proposed scheme is continuously below those of other two schemes, confirming better performance on MRI images compared to them.

scheme for both CT and MRI images under different attacks is much smaller than the values of S1, S2. These results indicate that the combination of RSC and IASV of our proposed scheme improves the overall watermarking performance.

Finally, we plot detection error tradeoff (DET) graphs in Fig. 9(d) and Fig. 10(d) to further demonstrate the superiority of our proposed scheme in terms of overall watermarking performance. We used 19900 points by calculating $P_{f n}$ with different $P_{f p}$ (from 1/19900 to 1 by increment of 1/19900) to draw the DET graphs. However, any point of which $P_{f n}$ equals to 0 would not appear in the DET graphs. The curves of our proposed scheme for both CT and MRI images are continuously below those of other two schemes, confirming better performance compared to them on both CT and MRI images. In particular, when the $P_{f p}$ is set to larger than $1 / 19900(2 / 19900)$ 
Table 7: Comparison of $P_{f n}$ with S1 and S2 under different attacks when $P_{f p}$ is set to $1 / 19900$ on CT images

\begin{tabular}{|c|c|c|c|}
\hline Attack & $\begin{array}{c}\mathrm{S} 1 \\
(\mathrm{IASV}+\text { Fan-ring })\end{array}$ & $\begin{array}{c}\mathrm{S} 2 \\
(\mathrm{RSC})\end{array}$ & $\begin{array}{l}\text { Proposed Scheme } \\
\quad(\mathrm{RSC}+\mathrm{IASV})\end{array}$ \\
\hline JC $30 \%$ & 0.0000 & 0.0100 & 0.0000 \\
\hline JC $50 \%$ & 0.0000 & 0.0000 & 0.0000 \\
\hline $\mathrm{CA}+20 \%$ & 0.0000 & 0.0150 & 0.0000 \\
\hline CA $-20 \%$ & 0.0000 & 0.0000 & 0.0000 \\
\hline $\mathrm{BA}+20 \%$ & 0.0000 & 0.0000 & 0.0000 \\
\hline BA $-20 \%$ & 0.0000 & 0.0000 & 0.0000 \\
\hline GN 0.01 & 0.0050 & 0.1100 & 0.0000 \\
\hline GN 0.02 & 0.1950 & 0.7000 & 0.0100 \\
\hline SPN 0.01 & 0.0000 & 0.2400 & 0.0000 \\
\hline SPN 0.02 & 0.0000 & 0.4900 & 0.0000 \\
\hline GF $3 \times 3$ & 0.0000 & 0.1000 & 0.0000 \\
\hline GF $5 \times 5$ & 0.0000 & 0.1050 & 0.0000 \\
\hline $\mathrm{AF} 3 \times 3$ & 0.0000 & 0.1000 & 0.0000 \\
\hline $\mathrm{AF} 5 \times 5$ & 0.0000 & 0.1250 & 0.0000 \\
\hline MF $3 \times 3$ & 0.0000 & 0.0950 & 0.0000 \\
\hline MF $5 \times 5$ & 0.0000 & 0.1200 & 0.0000 \\
\hline RS $50 \%$ & 0.0000 & 0.1000 & 0.0000 \\
\hline RS $200 \%$ & 0.0000 & 0.0550 & 0.0000 \\
\hline CIE $15 \%$ & 0.0000 & 0.2800 & 0.0000 \\
\hline CIE $20 \%$ & 0.0000 & 0.3100 & 0.0000 \\
\hline $\mathrm{SF}(\mathrm{H}) 1 \%$ & 0.1650 & 0.0150 & 0.0000 \\
\hline $\mathrm{SF}(\mathrm{V}) 1 \%$ & 0.4250 & 0.0950 & 0.0050 \\
\hline $\mathrm{RT} 45^{\circ}$ & 1.0000 & 0.0000 & 0.0000 \\
\hline $\mathrm{RT} 60^{\circ}$ & 1.0000 & 0.0000 & 0.0000 \\
\hline RT $90^{\circ}$ & 1.0000 & 0.0000 & 0.0000 \\
\hline $\mathrm{FL}(\mathrm{H})$ & 1.0000 & 0.0000 & 0.0000 \\
\hline $\mathrm{FL}(\mathrm{V})$ & 1.0000 & 0.0000 & 0.0000 \\
\hline Average: & 0.2144 & 0.1135 & 0.0006 \\
\hline
\end{tabular}

for CT (MRI) images, all the $P_{f n}$ by using our proposed scheme always equal to 0 while the $P_{f n}$ of other benchmarked methods are still higher than 0 . As a result, the number of points by using our proposed scheme which appear in the DET graphs is much smaller than those of the benchmarked methods. In addition, when the $P_{f p}$ is set to $1 / 19900$ (the smallest $P_{f p}$ except 0 in our experiment), the $P_{f n}$ of our proposed scheme is still lower than $10^{-3}$, while the values of other two schemes are higher than $10^{-1}$ for CT images and higher than $10^{-2}$ for MRI images.

The above experiments verify the effectiveness of our designed RSC and IASV. 
Table 8: Comparison of $P_{f n}$ with S1 and S2 under different attacks when $P_{f p}$ is set to $1 / 19900$ on MRI images

\begin{tabular}{|c|c|c|c|}
\hline Attack & $\begin{array}{c}\mathrm{S} 1 \\
(\mathrm{IASV}+\mathrm{Fan}-\text { ring })\end{array}$ & $\begin{array}{c}\mathrm{S} 2 \\
(\mathrm{RSC})\end{array}$ & $\begin{array}{l}\text { Proposed Scheme } \\
\quad(\mathrm{RSC}+\mathrm{IASV})\end{array}$ \\
\hline $\mathrm{JC} 30 \%$ & 0.0000 & 0.0000 & 0.0000 \\
\hline JC $50 \%$ & 0.0000 & 0.0000 & 0.0000 \\
\hline $\mathrm{CA}+20 \%$ & 0.0000 & 0.0000 & 0.0000 \\
\hline CA $-20 \%$ & 0.0000 & 0.0000 & 0.0000 \\
\hline $\mathrm{BA}+20 \%$ & 0.0000 & 0.0000 & 0.0000 \\
\hline $\mathrm{BA}-20 \%$ & 0.0000 & 0.0000 & 0.0000 \\
\hline GN 0.01 & 0.0000 & 0.0000 & 0.0000 \\
\hline GN 0.02 & 0.0000 & 0.0000 & 0.0000 \\
\hline SPN 0.01 & 0.0000 & 0.2600 & 0.0000 \\
\hline SPN 0.02 & 0.0000 & 0.3350 & 0.0100 \\
\hline $\mathrm{GF} 3 \times 3$ & 0.0000 & 0.0000 & 0.0000 \\
\hline GF $5 \times 5$ & 0.0000 & 0.0000 & 0.0000 \\
\hline $\mathrm{AF} 3 \times 3$ & 0.0000 & 0.0000 & 0.0000 \\
\hline $\mathrm{AF} 5 \times 5$ & 0.0000 & 0.0050 & 0.0000 \\
\hline $\mathrm{MF} 3 \times 3$ & 0.0000 & 0.0000 & 0.0000 \\
\hline $\mathrm{MF} 5 \times 5$ & 0.0000 & 0.0000 & 0.0000 \\
\hline RS 50\% & 0.0000 & 0.0000 & 0.0000 \\
\hline RS $200 \%$ & 0.0000 & 0.0000 & 0.0000 \\
\hline CIE $15 \%$ & 0.0000 & 0.0000 & 0.0000 \\
\hline CIE $20 \%$ & 0.0000 & 0.0400 & 0.0150 \\
\hline $\mathrm{SF}(\mathrm{H}) 1 \%$ & 0.7850 & 0.0000 & 0.0100 \\
\hline $\mathrm{SF}(\mathrm{V}) 1 \%$ & 0.8050 & 0.0000 & 0.0100 \\
\hline $\mathrm{RT} 45^{\circ}$ & 1.0000 & 0.0000 & 0.0000 \\
\hline $\mathrm{RT} 60^{\circ}$ & 1.0000 & 0.0000 & 0.0000 \\
\hline RT $90^{\circ}$ & 1.0000 & 0.0000 & 0.0000 \\
\hline $\mathrm{FL}(\mathrm{H})$ & 1.0000 & 0.0000 & 0.0000 \\
\hline $\mathrm{FL}(\mathrm{V})$ & 1.0000 & 0.0000 & 0.0000 \\
\hline Average: & 0.2441 & 0.0248 & 0.0017 \\
\hline
\end{tabular}

By combining the strength of the RSC and IASV, our proposed scheme achieves an optimal trade-off between distinguishability and robustness.

\subsection{Comparisons with other state-of-the-art zero-watermarking schemes}

In this section, we compare our proposed scheme with five state-of-the-art zerowatermarking schemes to evaluate the watermarking performance in terms of the distinguishability and robustness. They are named Hu-6 based Scheme [35], QPHTs based Scheme [37], Fan-ring based Scheme [40], 3D-DCT based Scheme [31] and 
Table 9: Comparison of the inter-BERs with the benchmarked schemes

\begin{tabular}{llcc}
\hline & Average & Minimum \\
\hline & Hu-6 based Scheme [35] & 0.0687 & 0.0000 \\
QPHTs based Scheme [37] & 0.1316 & 0.0381 \\
CT images & Fan-ring based Scheme [40] & 0.3845 & 0.1351 \\
& 3D-DCT based Scheme [31] & 0.3567 & 0.0000 \\
& 3D-DTCWT based Scheme [33] & 0.4682 & 0.0313 \\
& Proposed Scheme & 0.4428 & 0.2207 \\
\hline & Hu-6 based Scheme [35] & 0.0409 & 0.0000 \\
MRI images & Fan-ring based Scheme [40] & 0.1790 & 0.0498 \\
& 3D-DCT based Scheme [31] & 0.4187 & 0.2213 \\
& 3D-DTCWT based Scheme [33] & 0.4611 & 0.0469 \\
Proposed Scheme & 0.4870 & 0.0313 \\
\hline
\end{tabular}

3D-DTCWT based Scheme [33].

\subsubsection{Comparison of distinguishability}

First, we also use inter-BERs to evaluate the distinguishability of the proposed scheme. The average and minimum values of inter-BERs by using our proposed scheme and the benchmarked schemes are shown in Table 9. From Table 9, we can see that the average and minimum values of inter-BERs by using our proposed scheme are much larger than those of the benchmarked schemes. These results demonstrate that the distinguishability of our proposed scheme for both CT and MRI images is much higher than the benchmarked schemes. The reasons for this are as follows. Firstly, compared with methods of single slice only [35, 37, 40], the deployment of multi-slices makes better use of the temporal characteristics of VMI and thus leads to higher distinguishability. Secondly, compared with using equal-size partition, the setting of smaller size to the central circles, which are more texture-rich, reflects the characteristics of these central regions more saliently and thus also improves the distinguishability. Finally, the calculation of intra variations of statistical low-order moments further enhances the distinguishability of our designed feature.

\subsubsection{Comparison of robustness}

Then, we use intra-BERs to evaluate the robustness of the proposed scheme. The mean intra-BERs obtained by using our proposed scheme and the benchmarked schemes for both CT and MRI images are listed in Table 10 and Table 11. The results in Table 10 and Table 11 show that the average values of mean intra-BERs of our proposed scheme are smaller than those of the benchmarked schemes for both CT and MRI images. In particular, our proposed scheme outperforms Hu-6 based scheme under the cropping attacks, and the other three benchmarked schemes (i.e., Fan-ring based scheme, 3D-DCT based scheme and 3D-DTCWT based scheme), under the rotation and flipping attacks. These results demonstrate that our proposed scheme 
Table 10: Comparison of the mean intra-BERs between the original watermark and the extracted watermarks with the benchmarked schemes on $\mathrm{CT}$ images

\begin{tabular}{|c|c|c|c|c|c|c|}
\hline Attack & $\begin{array}{c}\mathrm{Hu}-6 \\
{[35]}\end{array}$ & $\begin{array}{c}\text { QPHTs } \\
{[37]}\end{array}$ & $\begin{array}{c}\text { Fan-ring } \\
{[40]}\end{array}$ & $\begin{array}{c}\text { 3D-DCT } \\
{[31]}\end{array}$ & $\begin{array}{c}\text { 3D-DTCWT } \\
{[33]}\end{array}$ & $\begin{array}{c}\text { Proposed } \\
\text { Scheme }\end{array}$ \\
\hline JC $30 \%$ & 0.3775 & 0.0156 & 0.0970 & 0.0038 & 0.0108 & 0.0284 \\
\hline JC $50 \%$ & 0.3077 & 0.0101 & 0.0758 & 0.0017 & 0.0067 & 0.0200 \\
\hline $\mathrm{CA}+20 \%$ & 0.3136 & 0.0251 & 0.0389 & 0.0271 & 0.0197 & 0.0304 \\
\hline CA $-20 \%$ & 0.1044 & 0.0612 & 0.0119 & 0.1070 & 0.0022 & 0.0024 \\
\hline $\mathrm{BA}+20 \%$ & 0.0010 & 0.0048 & 0.0071 & 0.0010 & 0.0017 & 0.0224 \\
\hline BA $-20 \%$ & 0.0107 & 0.0013 & 0.0123 & 0.0004 & 0.0020 & 0.0028 \\
\hline GN 0.01 & 0.0960 & 0.0632 & 0.1557 & 0.0363 & 0.0177 & 0.0940 \\
\hline GN 0.02 & 0.0960 & 0.0869 & 0.1799 & 0.0540 & 0.0214 & 0.1303 \\
\hline SPN 0.01 & 0.0497 & 0.0426 & 0.0997 & 0.0059 & 0.0094 & 0.0892 \\
\hline SPN 0.02 & 0.0753 & 0.0585 & 0.1261 & 0.0105 & 0.0138 & 0.1092 \\
\hline GF $3 \times 3$ & 0.0271 & 0.0129 & 0.0333 & 0.0007 & 0.0009 & 0.0382 \\
\hline GF $5 \times 5$ & 0.0468 & 0.0179 & 0.0394 & 0.0009 & 0.0011 & 0.0460 \\
\hline $\mathrm{AF} 3 \times 3$ & 0.0362 & 0.0155 & 0.0388 & 0.0008 & 0.0007 & 0.0411 \\
\hline $\mathrm{AF} 5 \times 5$ & 0.1184 & 0.0321 & 0.0614 & 0.0011 & 0.0019 & 0.0638 \\
\hline $\mathrm{MF} 3 \times 3$ & 0.0271 & 0.0116 & 0.0509 & 0.0012 & 0.0148 & 0.0354 \\
\hline MF $5 \times 5$ & 0.0982 & 0.0246 & 0.0709 & 0.0031 & 0.0188 & 0.0626 \\
\hline RS $50 \%$ & 0.0243 & 0.0923 & 0.0413 & 0.0006 & 0.0004 & 0.0371 \\
\hline RS $200 \%$ & 0.0012 & 0.0108 & 0.0140 & 0.0005 & 0.0003 & 0.0150 \\
\hline CIE $15 \%$ & 0.1241 & 0.0877 & 0.0026 & 0.0605 & 0.0663 & 0.0583 \\
\hline CIE $20 \%$ & 0.1868 & 0.0947 & 0.0125 & 0.0805 & 0.0913 & 0.0748 \\
\hline $\mathrm{SF}(\mathrm{H}) 1 \%$ & 0.0195 & 0.0970 & 0.1817 & 0.0125 & 0.0627 & 0.1099 \\
\hline $\mathrm{SF}(\mathrm{V}) 1 \%$ & 0.0192 & 0.1081 & 0.2063 & 0.0177 & 0.0000 & 0.1385 \\
\hline $\mathrm{RT} 45^{\circ}$ & 0.0519 & 0.0976 & 0.4238 & 0.2341 & 0.2217 & 0.0239 \\
\hline $\mathrm{RT} 60^{\circ}$ & 0.0464 & 0.0972 & 0.4188 & 0.2531 & 0.2647 & 0.0250 \\
\hline $\mathrm{RT} 90^{\circ}$ & 0.0155 & 0.0948 & 0.4137 & 0.2391 & 0.3047 & 0.0282 \\
\hline $\mathrm{FL}(\mathrm{H})$ & 0.0144 & 0.0577 & 0.3329 & 0.1057 & 0.5000 & 0.0282 \\
\hline $\mathrm{FL}(\mathrm{V})$ & 0.0068 & 0.0577 & 0.3962 & 0.2890 & 0.0000 & 0.0353 \\
\hline Average: & 0.0850 & 0.0511 & 0.1312 & 0.0574 & 0.0613 & 0.0515 \\
\hline
\end{tabular}

has stronger robustness, especially against geometric attacks. The reasons for this are threefold: (i) the use of intra-slice variations of statistical low-order moments ensures the robustness against non-geometric attacks, (ii) the deployment of our ring-based partition tactic guarantees the robustness against rotation and flipping attacks and improves the robustness against cropping attacks, and (iii) the utilize of preprocess step in our scheme further ensures the robustness against resize attacks. 
Table 11: Comparison of the mean intra-BERs between the original watermark and the extracted watermarks with the benchmarked schemes on MRI images

\begin{tabular}{|c|c|c|c|c|c|c|}
\hline Attack & $\begin{array}{c}\mathrm{Hu}-6 \\
{[35]}\end{array}$ & $\begin{array}{c}\text { QPHTs } \\
{[37]}\end{array}$ & $\begin{array}{c}\text { Fan-ring } \\
{[40]}\end{array}$ & $\begin{array}{c}\text { 3D-DCT } \\
{[31]}\end{array}$ & $\begin{array}{c}\text { 3D-DTCWT } \\
{[33]}\end{array}$ & $\begin{array}{c}\text { Proposed } \\
\text { Scheme }\end{array}$ \\
\hline JC $30 \%$ & 0.0560 & 0.0209 & 0.0416 & 0.0014 & 0.0055 & 0.0229 \\
\hline JC $50 \%$ & 0.0402 & 0.0141 & 0.0290 & 0.0010 & 0.0034 & 0.0149 \\
\hline $\mathrm{CA}+20 \%$ & 0.2160 & 0.0414 & 0.0338 & 0.0637 & 0.1875 & 0.0570 \\
\hline CA $-20 \%$ & 0.0285 & 0.0473 & 0.0096 & 0.0799 & 0.0011 & 0.0028 \\
\hline $\mathrm{BA}+20 \%$ & 0.0287 & 0.0120 & 0.0083 & 0.0114 & 0.0109 & 0.0199 \\
\hline BA $-20 \%$ & 0.0260 & 0.0012 & 0.0046 & 0.0003 & 0.0011 & 0.0028 \\
\hline GN 0.01 & 0.0210 & 0.0634 & 0.0870 & 0.0158 & 0.0214 & 0.0683 \\
\hline GN 0.02 & 0.0210 & 0.0860 & 0.1118 & 0.0262 & 0.0305 & 0.0975 \\
\hline SPN 0.01 & 0.0082 & 0.0411 & 0.0448 & 0.0035 & 0.0077 & 0.0835 \\
\hline SPN 0.02 & 0.0133 & 0.0585 & 0.0652 & 0.0066 & 0.0136 & 0.1018 \\
\hline $\mathrm{GF} 3 \times 3$ & 0.0074 & 0.0199 & 0.0210 & 0.0016 & 0.0011 & 0.0334 \\
\hline GF $5 \times 5$ & 0.0088 & 0.0282 & 0.0296 & 0.0019 & 0.0020 & 0.0464 \\
\hline $\mathrm{AF} 3 \times 3$ & 0.0083 & 0.0237 & 0.0254 & 0.0019 & 0.0017 & 0.0389 \\
\hline $\mathrm{AF} 5 \times 5$ & 0.0145 & 0.0491 & 0.0579 & 0.0037 & 0.0034 & 0.0773 \\
\hline $\mathrm{MF} 3 \times 3$ & 0.0142 & 0.0201 & 0.0251 & 0.0035 & 0.0045 & 0.0295 \\
\hline MF $5 \times 5$ & 0.0231 & 0.0429 & 0.0559 & 0.0070 & 0.0084 & 0.0654 \\
\hline RS $50 \%$ & 0.0084 & 0.0878 & 0.0232 & 0.0001 & 0.0014 & 0.0284 \\
\hline RS $200 \%$ & 0.0029 & 0.0307 & 0.0028 & 0.0001 & 0.0001 & 0.0027 \\
\hline CIE $15 \%$ & 0.1923 & 0.1052 & 0.0020 & 0.1444 & 0.1514 & 0.0544 \\
\hline CIE $20 \%$ & 0.2681 & 0.1131 & 0.0148 & 0.1782 & 0.1828 & 0.0923 \\
\hline $\mathrm{SF}(\mathrm{H}) 1 \%$ & 0.0080 & 0.1215 & 0.1805 & 0.0156 & 0.0337 & 0.1098 \\
\hline $\mathrm{SF}(\mathrm{V}) 1 \%$ & 0.0075 & 0.1223 & 0.1847 & 0.0167 & 0.0000 & 0.1326 \\
\hline $\mathrm{RT} 45^{\circ}$ & 0.1492 & 0.1353 & 0.4382 & 0.3388 & 0.2680 & 0.0258 \\
\hline $\mathrm{RT} 60^{\circ}$ & 0.1414 & 0.1352 & 0.4258 & 0.3593 & 0.2981 & 0.0277 \\
\hline $\mathrm{RT} 90^{\circ}$ & 0.0294 & 0.1189 & 0.4178 & 0.3392 & 0.3250 & 0.0311 \\
\hline $\mathrm{FL}(\mathrm{H})$ & 0.0156 & 0.0738 & 0.3703 & 0.1808 & 0.5000 & 0.0308 \\
\hline $\mathrm{FL}(\mathrm{V})$ & 0.0249 & 0.0738 & 0.4227 & 0.3055 & 0.0000 & 0.0375 \\
\hline Average: & 0.0512 & 0.0625 & 0.1161 & 0.0781 & 0.0765 & 0.0495 \\
\hline
\end{tabular}

\subsubsection{Comparison of overall performance}

Finally, we plot the histograms of inter-BER and intra-BER, calculate the false positive rate $P_{f p}$ and the false negative rate $P_{f n}$, and draw DET graphs by using 19900 points of $P_{f n}$ with different $P_{f p}$ (from 1/19900 to 1 by increment of 1/19900) to evaluate the overall watermarking performance of our proposed scheme.

Fig. 11 and Fig. 12 (a)-(f) show that the distribution of inter-BER and intraBER in the benchmarked schemes have certain amount of overlapped regions. On the contrary, the distribution of inter-BER and intra-BER in our proposed scheme 


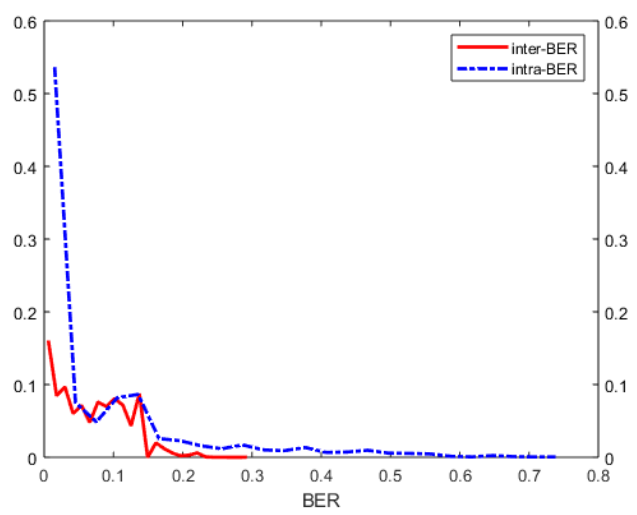

(a) Histograms of Hu-6 based Scheme

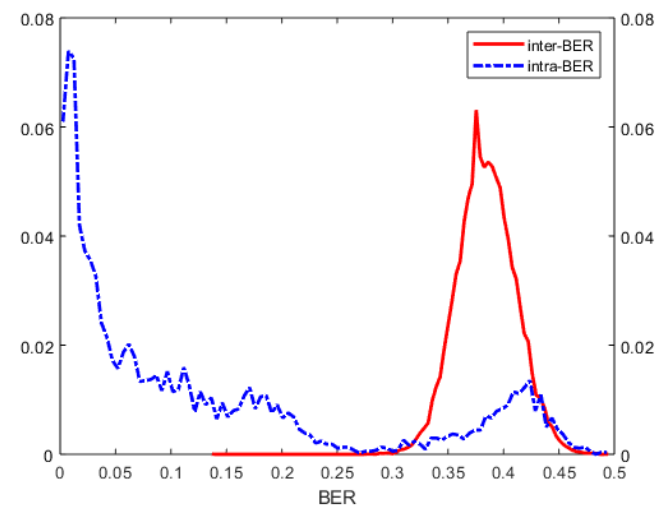

(c) Histograms of Fan-ring based Scheme

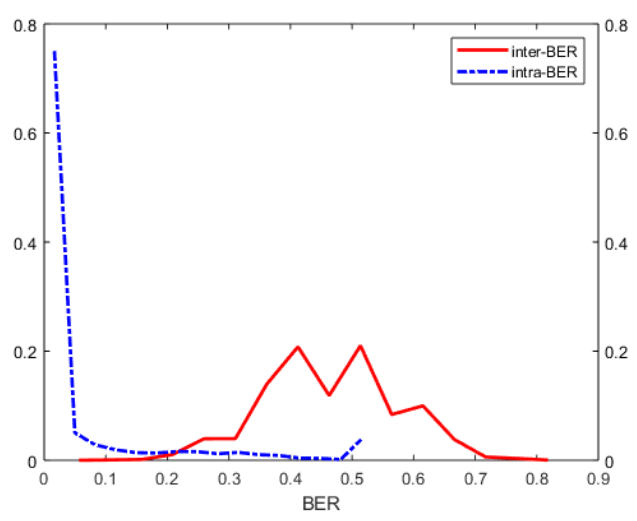

(e) Histograms of 3D-DTCWT based Scheme

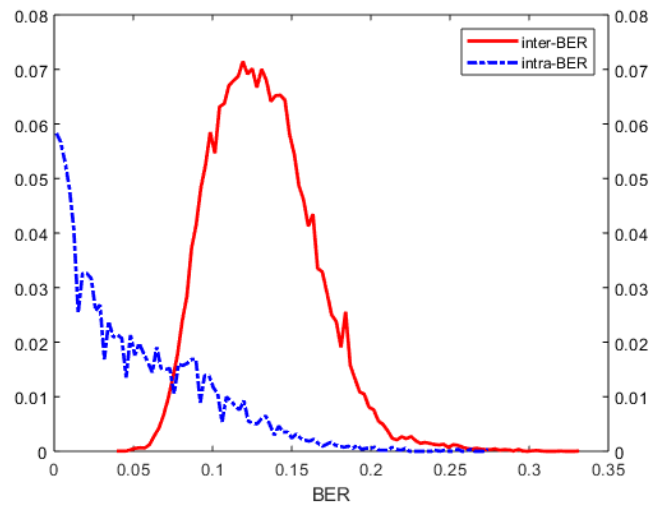

(b) Histograms of QPHTs based Scheme

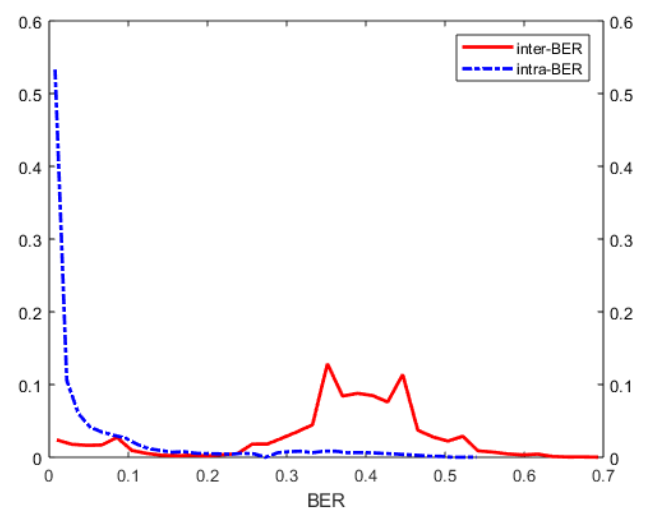

(d) Histograms of 3D-DCT based Scheme

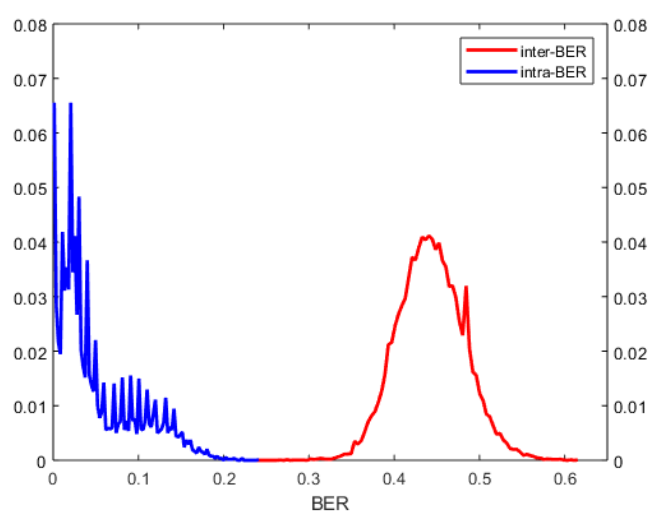

(f) Histograms of proposed Scheme

Fig. 11: (a)-(f) are the histograms of inter-BER and intra-BER of our proposed scheme and the benchmarked schemes on CT images. We can see that the distribution of inter-BER and intra-BER of our proposed scheme has insignificant overlapped region whereas the distribution of inter-BER and intra-BER in the benchmarked schemes have certain amount of overlapped regions.

has insignificant overlapped region. These results demonstrate that the overall watermarking performance of our proposed scheme in terms of both distinguishability and robustness is better than those of the benchmarked schemes. 


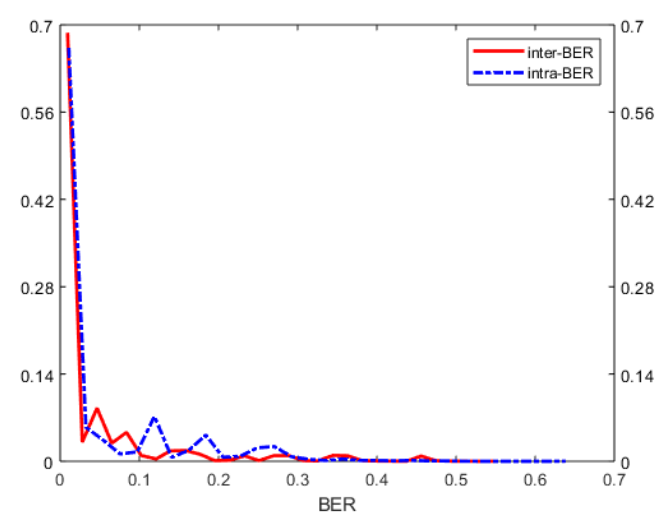

(a) Histograms of Hu-6 based Scheme

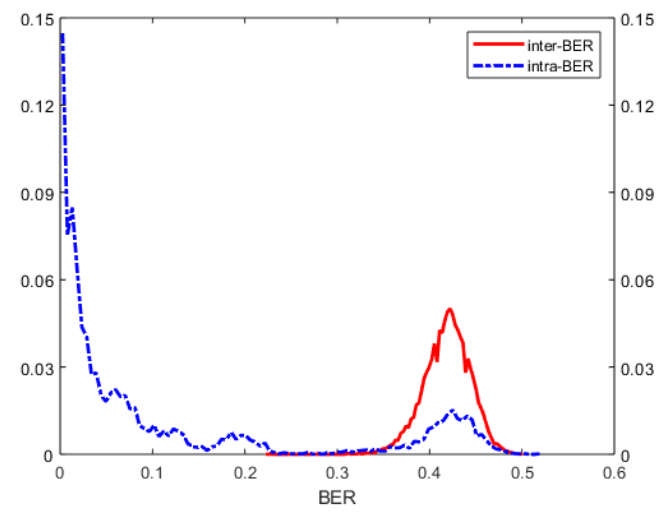

(c) Histograms of Fan-ring based Scheme

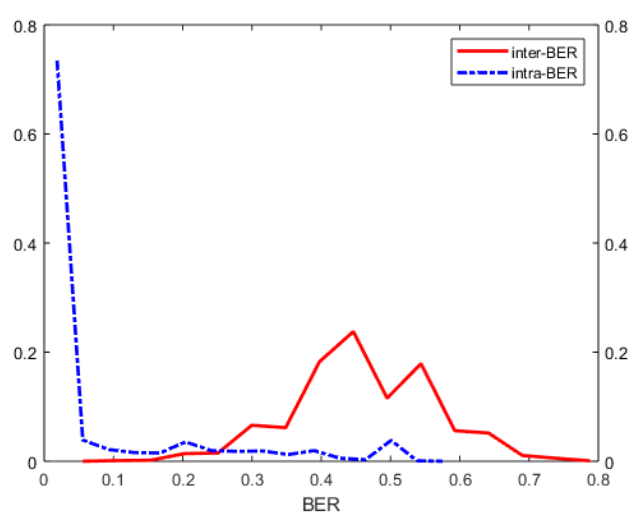

(e) Histograms of 3D-DTCWT based Scheme

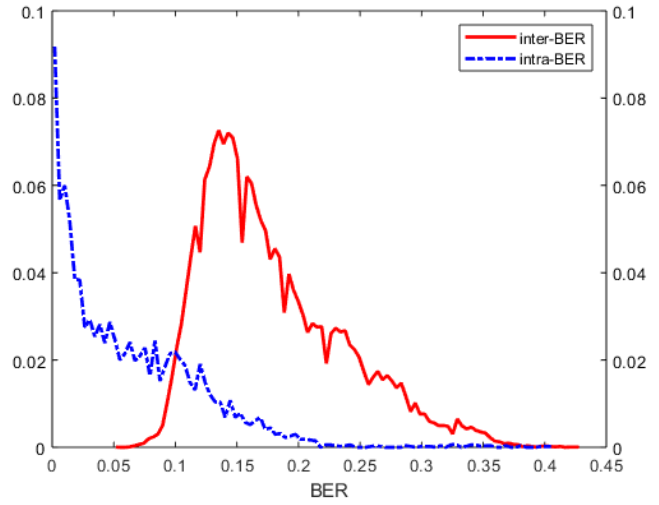

(b) Histograms of QPHTs based Scheme

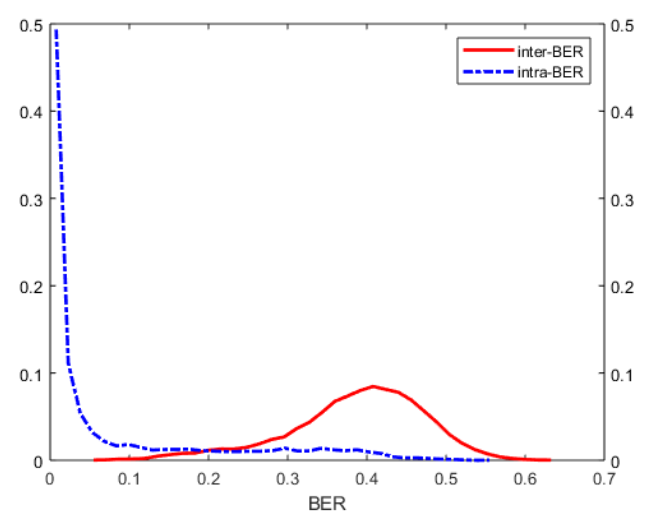

(d) Histograms of 3D-DCT based Scheme

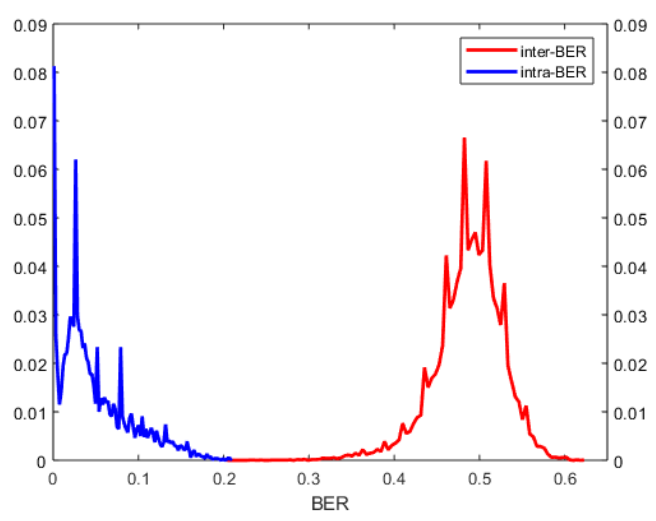

(f) Histograms of proposed Scheme

Fig. 12: (a)-(f) are the histograms of inter-BER and intra-BER of our proposed scheme and the benchmarked schemes on MRI images. We can see that the distribution of inter-BER and intra-BER of our proposed scheme has insignificant overlapped region whereas the distribution of inter-BER and intra-BER in the benchmarked schemes have certain amount of overlapped regions.

As shown in Table 12 and Table 13, when $P_{f p}$ is set to $1 / 19900$ (the smallest $P_{f p}$ except 0 in our experiment), the average value of $P_{f n}$ of our proposed scheme is much smaller than those of the benchmarked schemes for both CT and MRI images. 
More interestingly, the results of our proposed scheme are essentially perfect (close to 0 under all attacks) while all the five benchmarked schemes fail under a number of attacks. In particular, the $P_{f n}$ of the benchmarked schemes under the rotation and flipping attacks are unacceptable whereas the $P_{f_{n}}$ of our proposed scheme under the rotation and flipping attacks are equal to 0 by deploying our ring-based partition tactic. In addition, we also notice that the $P_{f n}$ of CT images are much higher than those of MRI images under noise addition attacks by using the Hu- 6 based scheme [35], QPHTs based scheme [37], Fan-ring based scheme [40], 3D-DCT based scheme [31]. The reason for this is that the CT images in ELCAP [46] and TCIA [47] have much less textures than the MRI images from Nottingham hospital database as shown in Fig. 8, which leads to worse distinguishability and robustness against the noise addition attacks for CT images by using these four benchmarked schemes. In the contrary, the $P_{f n}$ of our proposed scheme under noise addition attacks are close to 0 for both $\mathrm{CT}$ and MRI images by deploying the intra-slice variations of statistical low-order moments. These results demonstrate that our proposed scheme has the best overall watermarking performance by not only ensuring high feature distinguishability but also guaranteeing outstanding feature robustness.

As shown in Fig. 13, the curves of our proposed scheme is continuously below those of other five schemes for both CT and MRI images, confirming better performance on $\mathrm{CT}$ and MRI images compared to benchmarked schemes. In particular, when the $P_{f p}$ is set to larger than 1/19900 (2/19900) for CT (MRI) images, all the $P_{f n}$ by using our proposed scheme always equal to 0 while the $P_{f n}$ of other benchmarked methods are still much higher than 0 . As a result, the number of points by using our proposed scheme which appear in the DET graphs is much smaller than those of the benchmarked methods. In addition, when the $P_{f p}$ is set to $1 / 19900$ (the smallest $P_{f p}$ except 0 in our experiment), the $P_{f n}$ of our proposed scheme is still around $10^{-3}$, while the values of other schemes are higher than $10^{-1}$. These results demonstrate that our proposed scheme achieves a significantly better trade-off between distinguishability and robustness comparing to the benchmarked schemes.

\subsubsection{Comparison of computation complexity}

We evaluate the computational complexity of the different methods based on execution CPU timings of the different steps of both registration and identification phases for one CT and one MRI image. We execute this experiment on a computer with an Intel Core i5-7400 Processor (2 cores at $3.00 \mathrm{GHz}$ ) and 16G RAM with the implementations via Matlab. The results in terms of mean execution CPU timings over the $200 \mathrm{CT}$ and $200 \mathrm{MRI}$ images are listed in Table 14 and Table 15, respectively.

As we can see from Table 14 and Table 15, the mean total execution timings of our proposed method are only $0.83 \mathrm{~s}$ and $0.85 \mathrm{~s}$ for processing one CT image and one MRI image, respectively. These values are acceptable for processing one VMI with multiple slices. In particular, the execution timings for both phases are below those of QPHTs based scheme [37] (about 3\%-5\% of them), adding to the superiority of our method. In addition, our total execution timings are comparable with those of 3D-DTCWT based scheme [33], while our proposed method shows superiority in terms of both distinguishability and robustness as mentioned in sections 4.4.1 to 
Table 12: Comparison of $P_{f n}$ with the benchmarked schemes under different attacks when $P_{f p}$ is set to $1 / 19900$ on $\mathrm{CT}$ images

\begin{tabular}{|c|c|c|c|c|c|c|}
\hline Attack & $\begin{array}{c}\mathrm{Hu}-6 \\
{[35]}\end{array}$ & $\begin{array}{c}\text { QPHTs } \\
{[37]}\end{array}$ & $\begin{array}{c}\text { Fan-ring } \\
{[40]}\end{array}$ & $\begin{array}{c}\text { 3D-DCT } \\
{[31]}\end{array}$ & $\begin{array}{c}\text { 3D-DTCWT } \\
{[33]}\end{array}$ & $\begin{array}{c}\text { Proposed } \\
\text { Scheme }\end{array}$ \\
\hline $\mathrm{JC} 30 \%$ & 1.0000 & 0.0450 & 0.0850 & 0.1850 & 0.0800 & 0.0000 \\
\hline JC $50 \%$ & 1.0000 & 0.0000 & 0.0100 & 0.0900 & 0.0250 & 0.0000 \\
\hline $\mathrm{CA}+20 \%$ & 0.6800 & 0.1450 & 0.0050 & 0.7900 & 0.1550 & 0.0000 \\
\hline CA $-20 \%$ & 0.9300 & 0.8400 & 0.0000 & 0.8950 & 0.0000 & 0.0000 \\
\hline $\mathrm{BA}+20 \%$ & 0.0350 & 0.0000 & 0.0000 & 0.0650 & 0.0000 & 0.0000 \\
\hline $\mathrm{BA}-20 \%$ & 0.2050 & 0.0000 & 0.0000 & 0.0300 & 0.0000 & 0.0000 \\
\hline GN 0.01 & 0.9200 & 0.7600 & 0.7400 & 0.6900 & 0.1500 & 0.0000 \\
\hline GN 0.02 & 0.9200 & 0.9150 & 0.8750 & 0.7300 & 0.1700 & 0.0100 \\
\hline SPN 0.01 & 0.8600 & 0.5050 & 0.0550 & 0.2650 & 0.0600 & 0.0000 \\
\hline SPN 0.02 & 0.9050 & 0.7300 & 0.3550 & 0.4300 & 0.1000 & 0.0000 \\
\hline GF $3 \times 3$ & 0.6750 & 0.0050 & 0.0000 & 0.0300 & 0.0000 & 0.0000 \\
\hline GF $5 \times 5$ & 0.8100 & 0.0200 & 0.0000 & 0.0400 & 0.0000 & 0.0000 \\
\hline $\mathrm{AF} 3 \times 3$ & 0.7300 & 0.0250 & 0.0000 & 0.0350 & 0.0000 & 0.0000 \\
\hline $\mathrm{AF} 5 \times 5$ & 0.9850 & 0.3100 & 0.0000 & 0.0500 & 0.0000 & 0.0000 \\
\hline $\mathrm{MF} 3 \times 3$ & 0.3550 & 0.0400 & 0.0450 & 0.0600 & 0.1300 & 0.0000 \\
\hline MF $5 \times 5$ & 0.7050 & 0.1400 & 0.1150 & 0.1600 & 0.1350 & 0.0000 \\
\hline RS $50 \%$ & 0.6650 & 0.6200 & 0.0000 & 0.0200 & 0.0000 & 0.0000 \\
\hline RS $200 \%$ & 0.0800 & 0.0050 & 0.0000 & 0.0150 & 0.0000 & 0.0000 \\
\hline CIE $15 \%$ & 1.0000 & 0.9200 & 0.0000 & 0.9200 & 0.5650 & 0.0000 \\
\hline CIE $20 \%$ & 1.0000 & 0.9900 & 0.0000 & 0.9850 & 0.6900 & 0.0000 \\
\hline $\mathrm{SF}(\mathrm{H}) 1 \%$ & 0.7350 & 0.9950 & 0.9950 & 0.5800 & 0.5400 & 0.0000 \\
\hline $\mathrm{SF}(\mathrm{V}) 1 \%$ & 0.7250 & 1.0000 & 1.0000 & 0.6500 & 0.0000 & 0.0050 \\
\hline $\mathrm{RT} 45^{\circ}$ & 0.6300 & 1.0000 & 1.0000 & 1.0000 & 0.9700 & 0.0000 \\
\hline $\mathrm{RT} 60^{\circ}$ & 0.6000 & 1.0000 & 1.0000 & 1.0000 & 0.9850 & 0.0000 \\
\hline $\mathrm{RT} 90^{\circ}$ & 0.4250 & 1.0000 & 1.0000 & 1.0000 & 0.9850 & 0.0000 \\
\hline $\mathrm{FL}(\mathrm{H})$ & 0.4250 & 0.8350 & 1.0000 & 0.8900 & 1.0000 & 0.0000 \\
\hline $\mathrm{FL}(\mathrm{V})$ & 0.2900 & 0.8350 & 1.0000 & 1.0000 & 0.0000 & 0.0000 \\
\hline Average: & 0.6774 & 0.5067 & 0.3437 & 0.4669 & 0.2496 & 0.0006 \\
\hline
\end{tabular}

4.4.3.

Although Hu-6 based scheme [35], 3D-DCT based scheme [31] and Fan-ring based scheme [40] are faster than our method, they are vulnerable to geometrical attacks such as cropping and rotation. Moreover, the distinguishabilities for individual VMI by using Hu-6 based scheme and 3D-DCT based scheme are insufficient to guarantee the accurate identification of authenticity and copyright ownership. In the contrary, our proposed method ensures the remarkable robustness under these attacks and sufficient distinguishability at the same time, confirming a better trade-off between the 
Table 13: Comparison of $P_{f n}$ with the benchmarked schemes under different attacks when $P_{f p}$ is set to $1 / 19900$ on MRI images

\begin{tabular}{|c|c|c|c|c|c|c|}
\hline Attack & $\begin{array}{c}\mathrm{Hu}-6 \\
{[35]}\end{array}$ & $\begin{array}{c}\text { QPHTs } \\
{[37]}\end{array}$ & $\begin{array}{c}\text { Fan-ring } \\
{[40]}\end{array}$ & $\begin{array}{c}\text { 3D-DCT } \\
{[31]}\end{array}$ & $\begin{array}{c}\text { 3D-DTCWT } \\
{[33]}\end{array}$ & $\begin{array}{c}\text { Proposed } \\
\text { Scheme }\end{array}$ \\
\hline $\mathrm{JC} 30 \%$ & 0.6700 & 0.0600 & 0.0000 & 0.0000 & 0.0100 & 0.0000 \\
\hline JC $50 \%$ & 0.5550 & 0.0100 & 0.0000 & 0.0000 & 0.0000 & 0.0000 \\
\hline $\mathrm{CA}+20 \%$ & 1.0000 & 0.2450 & 0.0000 & 0.5400 & 0.3650 & 0.0000 \\
\hline CA $-20 \%$ & 0.3000 & 0.4400 & 0.0000 & 0.7300 & 0.0000 & 0.0000 \\
\hline $\mathrm{BA}+20 \%$ & 0.2900 & 0.0000 & 0.0000 & 0.0500 & 0.0800 & 0.0000 \\
\hline $\mathrm{BA}-20 \%$ & 0.2700 & 0.0000 & 0.0000 & 0.0000 & 0.0000 & 0.0000 \\
\hline GN 0.01 & 0.2650 & 0.5950 & 0.0000 & 0.0200 & 0.1450 & 0.0000 \\
\hline GN 0.02 & 0.2650 & 0.7950 & 0.0000 & 0.0800 & 0.2350 & 0.0000 \\
\hline SPN 0.01 & 0.2100 & 0.3250 & 0.0000 & 0.0000 & 0.0500 & 0.0000 \\
\hline SPN 0.02 & 0.2450 & 0.5350 & 0.0000 & 0.0000 & 0.0950 & 0.0100 \\
\hline $\mathrm{GF} 3 \times 3$ & 0.2550 & 0.1350 & 0.0000 & 0.0000 & 0.0000 & 0.0000 \\
\hline GF $5 \times 5$ & 0.2850 & 0.1900 & 0.0000 & 0.0000 & 0.0050 & 0.0000 \\
\hline $\mathrm{AF} 3 \times 3$ & 0.2750 & 0.1650 & 0.0000 & 0.0000 & 0.0050 & 0.0000 \\
\hline $\mathrm{AF} 5 \times 5$ & 0.3650 & 0.2700 & 0.0000 & 0.0050 & 0.0150 & 0.0000 \\
\hline $\mathrm{MF} 3 \times 3$ & 0.3000 & 0.1550 & 0.0000 & 0.0100 & 0.0200 & 0.0000 \\
\hline MF $5 \times 5$ & 0.4000 & 0.2250 & 0.0000 & 0.0250 & 0.0400 & 0.0000 \\
\hline RS 50\% & 0.2900 & 0.3600 & 0.0000 & 0.0000 & 0.0000 & 0.0000 \\
\hline RS $200 \%$ & 0.1350 & 0.1750 & 0.0000 & 0.0000 & 0.0000 & 0.0000 \\
\hline CIE $15 \%$ & 1.0000 & 0.9800 & 0.0000 & 0.9250 & 0.8850 & 0.0000 \\
\hline CIE $20 \%$ & 1.0000 & 0.9850 & 0.0000 & 0.9550 & 0.9250 & 0.0150 \\
\hline $\mathrm{SF}(\mathrm{H}) 1 \%$ & 0.2500 & 1.0000 & 0.0550 & 0.0200 & 0.2700 & 0.0100 \\
\hline $\mathrm{SF}(\mathrm{V}) 1 \%$ & 0.2950 & 0.9950 & 0.1150 & 0.0150 & 0.0000 & 0.0100 \\
\hline $\mathrm{RT} 45^{\circ}$ & 1.0000 & 1.0000 & 1.0000 & 1.0000 & 1.0000 & 0.0000 \\
\hline $\mathrm{RT} 60^{\circ}$ & 1.0000 & 1.0000 & 1.0000 & 1.0000 & 1.0000 & 0.0000 \\
\hline $\mathrm{RT} 90^{\circ}$ & 0.2500 & 1.0000 & 1.0000 & 1.0000 & 0.9950 & 0.0000 \\
\hline $\mathrm{FL}(\mathrm{H})$ & 0.2700 & 0.9300 & 1.0000 & 0.9800 & 1.0000 & 0.0000 \\
\hline $\mathrm{FL}(\mathrm{V})$ & 0.3350 & 0.9300 & 1.0000 & 1.0000 & 0.0000 & 0.0000 \\
\hline Average: & 0.4361 & 0.5000 & 0.1915 & 0.3094 & 0.2644 & 0.0017 \\
\hline
\end{tabular}

protection performance and computation complexity than these three benchmarked methods.

\subsection{Subjective evaluation}

We display the watermarks recovered from an example CT and an example MRI in our experiments under various attacks for subjective evaluation of our proposed scheme. The results are listed in Fig. 14 and Fig. 15. We can clearly observe that the recovered watermarks of our proposed scheme are perceptually similar to 


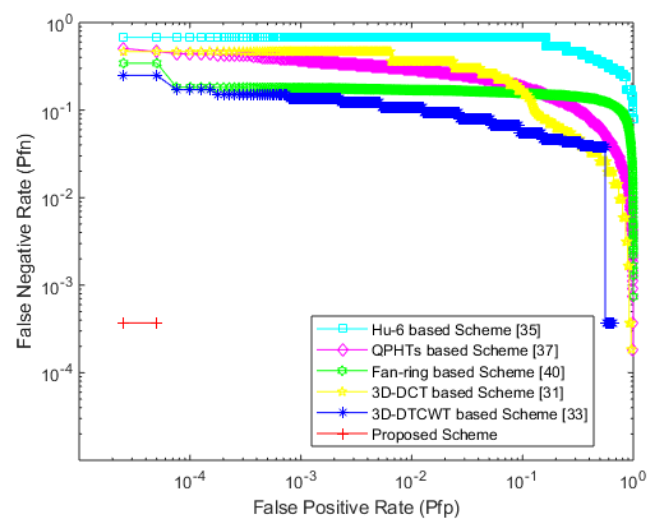

(a) DET graphs on CT images

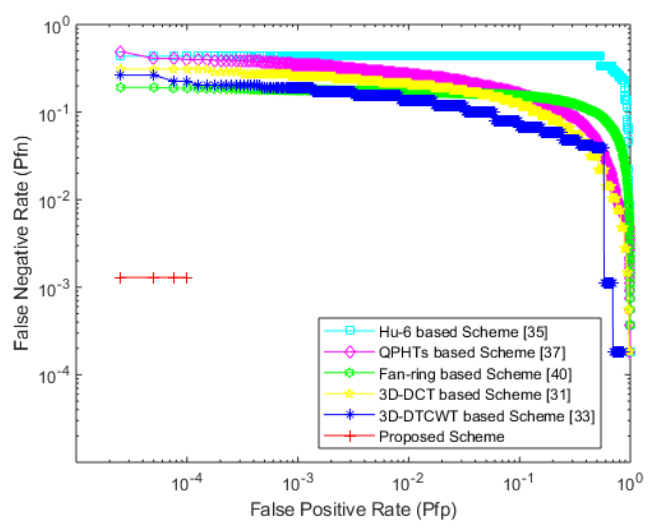

(b) DET graphs on MRI images

Fig. 13: (a) DET graphs of our proposed scheme and the benchmarked schemes on CT images. (b) DET graphs of our proposed scheme and the benchmarked schemes on MRI images. Note that any point of which $P_{f n}$ equals to 0 with different $P_{f p}$ (from 1/19900 to 1 by increment of 1/19900) would not appear in the DET graphs. As shown in (a) and (b), the curves of our proposed scheme are continuously below those of the benchmarked schemes, confirming better performance compared to them.

Table 14: Comparison of execution time with the benchmarked schemes on CT images

\begin{tabular}{|c|c|c|c|c|c|c|}
\hline & \multicolumn{3}{|c|}{ Registration phase } & \multicolumn{3}{|c|}{ Identification phase } \\
\hline & $\begin{array}{c}\text { Feature } \\
\text { extraction }\end{array}$ & $\begin{array}{c}\text { Generation of } \\
\text { ownership share }\end{array}$ & $\begin{array}{l}\text { Total } \\
\text { time }\end{array}$ & $\begin{array}{c}\text { Feature } \\
\text { extraction }\end{array}$ & $\begin{array}{c}\text { Copyright } \\
\text { identification }\end{array}$ & $\begin{array}{l}\text { Total } \\
\text { time }\end{array}$ \\
\hline$[35]$ & $1.47 \times 10^{-1}$ & $5.14 \times 10^{-2}$ & $1.99 \times 10^{-1}$ & $1.47 \times 10^{-1}$ & $4.42 \times 10^{-2}$ & $1.91 \times 10^{-1}$ \\
\hline$[37]$ & $2.85 \times 10^{1}$ & $2.51 \times 10^{-5}$ & $2.85 \times 10^{1}$ & $2.85 \times 10^{1}$ & $2.31 \times 10^{-5}$ & $2.85 \times 10^{1}$ \\
\hline$[40]$ & $5.97 \times 10^{-2}$ & $5.44 \times 10^{-3}$ & $6.51 \times 10^{-2}$ & $5.97 \times 10^{-2}$ & $5.36 \times 10^{-3}$ & $6.51 \times 10^{-2}$ \\
\hline$[31]$ & $4.07 \times 10^{-1}$ & $9.40 \times 10^{-5}$ & $4.07 \times 10^{-1}$ & $4.07 \times 10^{-1}$ & $9.80 \times 10^{-5}$ & $4.07 \times 10^{-1}$ \\
\hline$[33]$ & $6.97 \times 10^{-1}$ & $3.70 \times 10^{-5}$ & $6.97 \times 10^{-1}$ & $6.97 \times 10^{-1}$ & $8.00 \times 10^{-5}$ & $6.97 \times 10^{-1}$ \\
\hline Ours & $8.25 \times 10^{-1}$ & $4.67 \times 10^{-3}$ & $8.30 \times 10^{-1}$ & $8.25 \times 10^{-1}$ & $5.43 \times 10^{-3}$ & $8.30 \times 10^{-1}$ \\
\hline
\end{tabular}

Table 15: Comparison of execution time with the benchmarked schemes on MRI images

\begin{tabular}{|c|c|c|c|c|c|c|}
\hline & \multicolumn{3}{|c|}{ Registration phase } & \multicolumn{3}{|c|}{ Identification phase } \\
\hline & $\begin{array}{c}\text { Feature } \\
\text { extraction }\end{array}$ & $\begin{array}{c}\text { Generation of } \\
\text { ownership share }\end{array}$ & $\begin{array}{l}\text { Total } \\
\text { time }\end{array}$ & $\begin{array}{c}\text { Feature } \\
\text { extraction }\end{array}$ & $\begin{array}{c}\text { Copyright } \\
\text { identification }\end{array}$ & $\begin{array}{l}\text { Total } \\
\text { time }\end{array}$ \\
\hline$[35]$ & $1.03 \times 10^{-1}$ & $5.54 \times 10^{-2}$ & $1.58 \times 10^{-1}$ & $1.03 \times 10^{-1}$ & $4.32 \times 10^{-2}$ & $1.46 \times 10^{-1}$ \\
\hline$[37]$ & $1.67 \times 10^{1}$ & $2.42 \times 10^{-5}$ & $1.67 \times 10^{1}$ & $1.67 \times 10^{1}$ & $2.90 \times 10^{-5}$ & $1.67 \times 10^{1}$ \\
\hline$[40]$ & $6.64 \times 10^{-2}$ & $6.12 \times 10^{-3}$ & $7.25 \times 10^{-2}$ & $6.64 \times 10^{-2}$ & $4.51 \times 10^{-3}$ & $7.09 \times 10^{-2}$ \\
\hline$[31]$ & $5.68 \times 10^{-1}$ & $6.60 \times 10^{-5}$ & $5.68 \times 10^{-1}$ & $5.68 \times 10^{-1}$ & $6.40 \times 10^{-5}$ & $5.68 \times 10^{-1}$ \\
\hline$[33]$ & $6.62 \times 10^{-1}$ & $4.30 \times 10^{-5}$ & $6.62 \times 10^{-1}$ & $6.62 \times 10^{-1}$ & $8.20 \times 10^{-5}$ & $6.62 \times 10^{-1}$ \\
\hline Ours & $8.45 \times 10^{-1}$ & $4.59 \times 10^{-3}$ & $8.50 \times 10^{-1}$ & $8.45 \times 10^{-1}$ & $5.05 \times 10^{-3}$ & $8.50 \times 10^{-1}$ \\
\hline
\end{tabular}

the original watermark. These results indicate that our proposed scheme visually provide effective protection for VMIs by verifying the recovered watermarks. 


\begin{tabular}{|c|c|c|c|c|c|}
\hline Attack & $\begin{array}{l}\text { Proposed } \\
\text { Scheme }\end{array}$ & Attack & $\begin{array}{c}\text { Proposed } \\
\text { Scheme }\end{array}$ & Attack & $\begin{array}{c}\text { Proposed } \\
\text { Scheme }\end{array}$ \\
\hline $\mathrm{JC} 30 \%$ & & $\mathrm{JC} 50 \%$ & & $\mathrm{CA}+20 \%$ & \\
\hline CA $-20 \%$ & & $\mathrm{BA}+20 \%$ & & $\mathrm{BA}-20 \%$ & \\
\hline GN 0.01 & & GN 0.02 & 酷 & SPN 0.01 & \\
\hline SPN 0.02 & & GF $3 \times 3$ & & GF $5 \times 5$ & \\
\hline $\mathrm{AF} 3 \times 3$ & & $\mathrm{AF} 5 \times 5$ & & $\mathrm{MF} 3 \times 3$ & \\
\hline MF $5 \times 5$ & & RS $50 \%$ & & RS $200 \%$ & \\
\hline CIE $15 \%$ & & CIE $20 \%$ & & $\mathrm{SF}(\mathrm{H}) 1 \%$ & \\
\hline $\mathrm{SF}(\mathrm{V}) 1 \%$ & & $\mathrm{RT} 45^{\circ}$ & & $\mathrm{RT} 60^{\circ}$ & \\
\hline $\mathrm{RT} 90^{\circ}$ & & $\mathrm{FL}(\mathrm{H})$ & & $\mathrm{FL}(\mathrm{V})$ & \\
\hline
\end{tabular}

Fig. 14: Examples of recovered watermarks from CT images under different attacks

\subsection{Security Analysis}

In this section, we evaluate the security performance of our proposed method by deploying the LLS. We first calculated the BERs between our extracted features and the encrypted features after XOR function with binarized chaotic sequences under different initial values, $h_{0}$, from 0.4 to 0.5 with an increment of 0.0005 , namely encryption-BER, to evaluate the correlation between extracted features and the encrypted features, which is the fundamental aspect for a encryption function. An extracted feature and its encrypted format are considered to be independent when the encryption-BER is close to 0.5. In addition, we also tested the BERs between different binarized chaotic sequences under different initial values, $h_{0}$, from 0.4 to 0.5 with an increment of 0.0005 , namely key-sensitive-BER, to evaluate the sensitivity 


\begin{tabular}{|c|c|c|c|c|c|}
\hline Attack & $\begin{array}{c}\text { Proposed } \\
\text { Scheme }\end{array}$ & Attack & $\begin{array}{c}\text { Proposed } \\
\text { Scheme }\end{array}$ & Attack & $\begin{array}{c}\text { Proposed } \\
\text { Scheme }\end{array}$ \\
\hline $\mathrm{JC} 30 \%$ & & JC $50 \%$ & & $\mathrm{CA}+20 \%$ & \\
\hline CA $-20 \%$ & & $\mathrm{BA}+20 \%$ & & BA $-20 \%$ & \\
\hline GN 0.01 & & GN 0.02 & & SPN 0.01 & \\
\hline SPN 0.02 & & GF $3 \times 3$ & & GF $5 \times 5$ & \\
\hline $\mathrm{AF} 3 \times 3$ & & $\mathrm{AF} 5 \times 5$ & & $\mathrm{MF} 3 \times 3$ & \\
\hline MF $5 \times 5$ & & RS $50 \%$ & & RS $200 \%$ & \\
\hline CIE $15 \%$ & & CIE $20 \%$ & & $\mathrm{SF}(\mathrm{H}) 1 \%$ & \\
\hline $\mathrm{SF}(\mathrm{V}) 1 \%$ & & $\mathrm{RT} 45^{\circ}$ & & $\mathrm{RT} 60^{\circ}$ & \\
\hline RT $90^{\circ}$ & & $\mathrm{FL}(\mathrm{H})$ & & $\mathrm{FL}(\mathrm{V})$ & \\
\hline
\end{tabular}

Fig. 15: Examples of recovered watermarks from MRI images under different attacks

to tiny key differences, which is also important for an encryption function. The key sensitivity is considered to be remarkable when the key-sensitive-BERs between two different binarized chaotic sequences under two keys with tiny differences is close to 0.5. The security analysis results are shown in Table 16.

Table 16: Security analysis

\begin{tabular}{lccc}
\hline & Maximum & Average & Minimum \\
\hline Encryption-BER & 0.5293 & 0.5005 & 0.4736 \\
Key-sensitive-BER & 0.5732 & 0.4999 & 0.4385 \\
\hline
\end{tabular}


As shown in Table 16, both the average values of encryption-BERs and keysensitive-BERs are $\sim 0.5$ with their maximum and minimum values still close to 0.5 , which confirms the remarkable security performances of our proposed method by deploying the LLS.

\section{Conclusion}

In this paper, a novel zero-watermarking scheme with enhanced distinguishability and robustness for authenticity and copyright protection of VMI is proposed. In this scheme, ring statistics are deployed to guarantee both the watermarking distinguishability and robustness. In addition, we design an intra-slice variation based mechanism to further enhance the watermarking distinguishability. Last but not least, a logistic-logistic system based chaotic map is used to ensure the watermarking security. The experimental and analytical results demonstrate that our proposed scheme has the following advantages. Firstly, the proposed scheme guarantees the outstanding distinguishability for different VMIs. Secondly, the proposed scheme ensures the strong watermarking robustness, especially against strong geometric attacks, such as large-degree rotations and cropping. Finally, the entire process of our proposed scheme is lossless, which satisfies the requirement of unbiased diagnosis. In future work, we will explore how to use the proposed technology for protecting other types of digital images and for content-based image retrieval.

\section{Acknowledgment}

This research is supported by the National Natural Science Foundations of China [61602527, 61772555, 61772553], Natural Science Foundations of Hunan Provincial [2020JJ4746, 2017JJ3416, 2018JJ2548], and the State Scholarship Fund offered by China Scholarship Council, under Grant 201806375002.

\section{References}

[1] P. Schelkens, A. Munteanu, J. Barbarien, Wavelet Coding of Volumetric Medical Datasets, IEEE TRANSACTIONS ON MEDICAL IMAGING 22 (3) (2003) 441-458.

[2] L. Wang, X. Tang, J. Zhang, Statistical Property Guided Feature Extraction for Volume Data, IEICE Transactions on Information and Systems 101 (1) (2018) 261-264.

[3] J. J. Erasmus, S. B. Sabloff, CT, Positron Emission Tomography, and MRI in Staging Lung Cancer, CLINICS IN CHEST MEDICINE 29 (1) (2008) 39-57.

[4] D. Millon, D. Byl, P. Collard, Could new reconstruction CT techniques challenge MRI for the detection of brain metastases in the context of initial lung cancer staging?, European Radiology 28 (2) (2017) 1-10. 
[5] J. P. Laissy, P. M. Trotot, Post-pneumonectomy evaluation of the chest: A prospective comparative study of MRI with CT, Magnetic resonance imaging 7 (1) (1989) 55-60.

[6] A. Pezeshk, S. Hamidian, N. Petrick, 3-D Convolutional Neural Networks for Automatic Detection of Pulmonary Nodules in Chest CT, IEEE Journal of Biomedical and Health Informatics pp (99) (2018) 1-1.

[7] O. Pelkonen, T. Tikkakoski, J. Pyhtinen, Cerebral CT and MRI Findings in Cervicocephalic Artery Dissection, Acta Radiologica 45 (3) (2004) 259-265.

[8] P. Issar, S. Chinna, S. K. Issar, Evaluation of Cerebral Venous Thrombosis by CT, MRI and MR Venography, The Journal of the Association of Physicians of India 65 (11) (2017) 16-21.

[9] M. S. Khan, V. H. Mankar, G. Prashanthi, Skeletonization of 3D images using 2.5D and 3D algorithms, in: International Conference on Next Generation Computing Technologies, IEEE, 2016.

[10] N. A. Memon, S. A. M. Gilani, A. Ali, Watermarking of chest CT scan medical images for content authentication, in: International Conference on Information \& Communication Technologies, IEEE, 2011.

[11] H. Nyeem, W. Boles, C. Boyd, Utilizing Least Significant Bit-Planes of RONI Pixels for Medical Image Watermarking, in: Digital Image Computing: Techniques and Applications (DICTA), IEEE, 2013.

[12] R. Thanki, S. Borra, V. Dwivedi, A RONI Based Visible Watermarking Approach for Medical Image Authentication, Journal of Medical Systems 41 (9) (2017) 143 .

[13] S. M. Mousavi, A. Naghsh, A. A. Manaf, A robust medical image watermarking against salt and pepper noise for brain MRI images, Multimedia Tools and Applications 76 (7) (2017) 10313-10342.

[14] R. A. Ur, S. Kiran, M. Dhiaa, Robust and Fragile Medical Image Watermarking: A Joint Venture of Coding and Chaos Theories, Journal of Healthcare Engineering 2018 (2018) 1-11.

[15] A. Sharifara, A. Ghaderi, Medical Image Watermarking using 2D-DWT with Enhanced security and capacity, in: Journal of the ACM, 2017.

[16] S. Maheshkar, Region-based hybrid medical image watermarking for secure telemedicine applications, Kluwer Academic Publishers 76 (3) (2017) 3617-3647.

[17] N. Solanki, S. K. Malik, S. Chhikara, RONI Medical Image Watermarking using DWT and RSA, International Journal of Computer Applications 96 (9) (2014) 30-35. 
[18] K. Swaraja, Medical image region based watermarking for secured telemedicine, Multimedia Tools and Applications 77 (21) (2018) 28249-28280.

[19] H. Golpîra, H. Danyali, Reversible Blind Watermarking for Medical Images Based on Wavelet Histogram Shifting, in: IEEE International Symposium on Signal Processing \& Information Technology, IEEE, 2011, pp. 31-36.

[20] S. A. Parah, F. Ahad, J. A. Sheikh, A New Reversible and high capacity data hiding technique for E-healthcare applications, Multimedia Tools and Applications 76 (3) (2017) 3943-3975.

[21] A. F. Qasim, R. Aspin, F. Meziane, ROI-based reversible watermarking scheme for ensuring the integrity and authenticity of DICOM MR images, Multimedia Tools and Applications 78 (12) (2019) 16433.

[22] M. Arsalan, A. S. Qureshi, A. Khan, Protection of medical images and patient related information in healthcare: Using an intelligent and reversible watermarking technique, Applied Soft Computing 51 (2017) 168-179.

[23] S. Priya, B. Santhi, P. Swaminathan, Hybrid Transform Based Reversible Watermarking Technique for Medical Images in Telemedicine Applications, Optik - International Journal for Light and Electron Optics 145 (2017) 655-671.

[24] M. Turuk, A. Dhande, A Novel Texture-Quantization-Based Reversible Multiple Watermarking Scheme Applied to Health Information System, Journal of Digital Imaging 31 (2) (2017) 167-177.

[25] K. Balasamy, S. Ramakrishnan, An intelligent reversible watermarking system for authenticating medical images using Wavelet and PSO, Cluster Computing 22 (2) (2018) 4431-4442.

[26] B. Lei, E. L. Tan, S. Chen, Reversible watermarking scheme for medical image based on differential evolution, Expert Systems with Applications 41 (7) (2014) 3178-3188.

[27] R. Thabit, B. E. Khoo, A new robust lossless data hiding scheme and its application to color medical images, Digital Signal Processing 38 (2015) 77-94.

[28] I. A. Ansari, M. Pant, C. W. Ahn, Artificial bee colony optimized robustreversible image watermarking, Multimedia Tools and Applications 76 (17) (2017) 18001-18025.

[29] X. Liu, J. Lou, H. Fang, A novel robust reversible watermarking scheme for protecting authenticity and integrity of medical images, IEEE Access 7 (2019) 2169-3536.

[30] A. Singh, M. K. Dutta, A robust zero-watermarking scheme for teleophthalmological applications, Journal of King Saud University - Computer and Information Sciences 2017 (2017) S1319157817302951. 
[31] B. Han, J. Li, Y. Li, Zero-watermarking Algorithm for Medical Volume Data Based on Difference Hashing, International Journal of Computers, Communications \& Control 10 (2) (2015) 188.

[32] B. Han, J. Li, Medical Image Watermarking in Sub-block Three-dimensional Discrete Cosine Transform Domain, International Journal Bioautomotion 20 (1) (2016) 69-78.

[33] J. Liu, J. Ma, J. Li, M. Huang, N. Sadiq, Y. Ai, Robust Watermarking Algorithm for Medical Volume Data in Internet of Medical Things, IEEE Access 8 (2020) 93939-93961.

[34] T. Y. Fan, H. C. Chao, B. C. Chieu, Lossless medical image watermarking method based on significant difference of cellular automata transform coefficient, Signal Processing: Image Communication 70 (2019) 174-183.

[35] S. Vellaisamy, V. Ramesh, Inversion attack resilient zero-watermarking scheme for medical image authentication, IET Image Processing 8 (12) (2014) 718-727.

[36] S. Vellaisamy, V. Ramesh, A QR code based zero-watermarking scheme for authentication of medical images in teleradiology cloud, Computational and Mathematical Methods in Medicine 2013 (2013) 1-16.

[37] Z. Xia, X. Wang, W. Zhou, Color medical image lossless watermarking using chaotic system and accurate quaternion polar harmonic transforms, Signal Processing 157 (2019) 108-118.

[38] Z. Xia, X. Wang, M. Wang, Geometrically Invariant Color Medical Image NullWatermarking Based on Precise Quaternion Polar Harmonic Fourier Moments, IEEE Access 7 (2019) 122544-122560.

[39] X. Liu, J. Lou, Y. Wang, Discriminative and robust zero-watermarking scheme based on completed local binary pattern for authentication and copyright identification of medical images, in: Imaging Informatics for Healthcare, Research, \& Applications, 2018, p. 54.

[40] B. Zou, J. Du, X. Liu, Distinguishable zero-watermarking scheme with similarity-based retrieval for digital rights Management of Fundus Image, Multimedia Tools and Applications 77 (8) (2018) 1-24.

[41] H. Liu, D. Xiao, R. Zhang, Y. Zhang, S. Bai, Robust and hierarchical watermarking of encrypted images based on compressive sensing, Signal Processing: Image Communication 45 (2016) 41-51.

[42] Z. Shao, Y. Shang, R. Zeng, H. Shu, G. Coatrieux, J. Wu, Robust watermarking scheme for color image based on quaternion-type moment invariants and visual cryptography, Signal Processing: Image Communication 48 (2016) 12-21. 
[43] Y. Bai, G. Jiang, M. Yu, Z. Peng, F. Chen, Towards a tone mapping-robust watermarking algorithm for high dynamic range image based on spatial activity, Signal Processing: Image Communication 65 (2018) 187-200.

[44] C. Pak, L. Huang, A new color image encryption using combination of the 1D chaotic map, Signal Processing 138 (2017) 129-137.

[45] M. Naor, A. Shamir, Visual cryptography, in: Workshop on the Theory and Application of of Cryptographic Techniques, Springer, 1994, pp. 1-12.

[46] A. P. Reeves, Y. Xie, S. Liu, Large-scale image region documentation for fully automated image biomarker algorithm development and evaluation, Journal of Medical Imaging 4 (2) (2017) 024505.

[47] H. Roth, L. Lu, A. Seff, A new 2.5 D representation for lymph node detection in CT, The Cancer Imaging Archive.

[48] E. Michalopoulou, A. Gale, Y. Chen, Prostate Imaging Self-assessment and Mentoring (PRISM): a prototype self-assessment scheme, in: Medical Imaging 2019: Image Perception, Observer Performance, and Technology Assessment. International Society for Optics and Photonics, Vol. 10952, 2019, p. 1095212.

[49] T.-S. Chen, J. Chen, J.-G. Chen, A simple and efficient watermark technique based on jpeg2000 codec, Multimedia systems 10 (1) (2004) 16-26.

[50] A. Sverdlov, S. Dexter, A. M. Eskicioglu, Robust det-svd domain image watermarking for copyright protection: embedding data in all frequencies, in: 2005 13th European Signal Processing Conference, IEEE, 2005, pp. 1-4.

[51] S.-C. Byun, I. L. Lee, T. H. Shin, B.-H. Ahn, A public-key based watermarking for color image authentication, in: Proceedings. IEEE International Conference on Multimedia and Expo, Vol. 1, IEEE, 2002, pp. 593-596. 


\section{Declaration of interests}

$\bigotimes$ The authors declare that they have no known competing financial interests or personal relationships that could have appeared to influence the work reported in this paper.

$\square$ The authors declare the following financial interests/personal relationships which may be considered as potential competing interests:

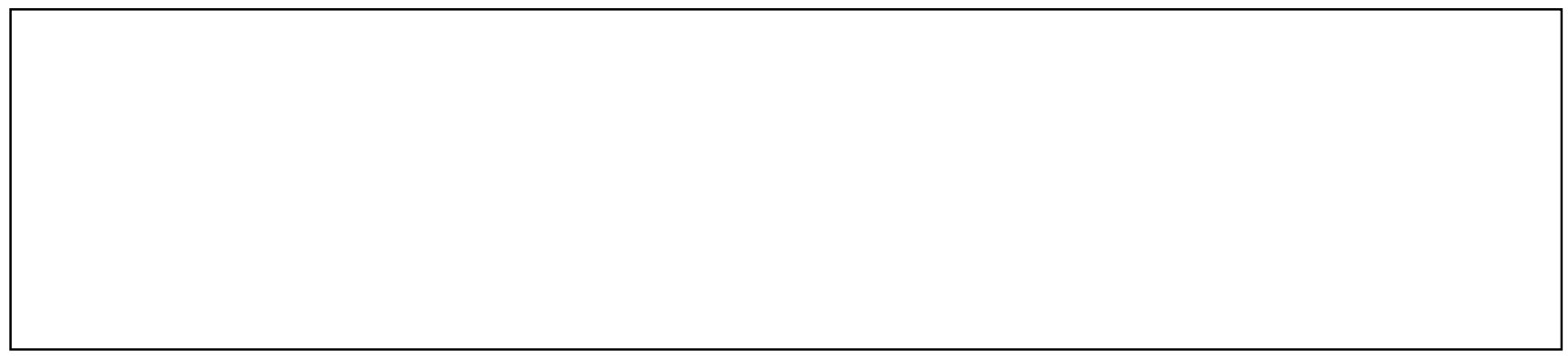


Dear Editors:

The paper was co-authored by Xiyao Liu, Yuying Sun, Jiahui Wang, Cundian Yang, Yayun Zhang, Lei Wang, Yan Chen, Hui Fang. All authors have contributed significantly for the paper as described below.

Xiyao Liu: Conceptualization, Methodology, Formal analysis, Investigation, Resources, Data Curation, Writing - Original Draft, Writing - Review \& Editing, Visualization, Supervision, Project administration, Funding acquisition. Yuying Sun: Methodology, Software, Validation, Investigation, Data Curation, Writing - Original Draft, Writing - Review \& Editing. Jiahui Wang: Software, Validation, Formal analysis, Writing - Review \& Editing. Cundian Yang: Software, Validation. Yayun Zhang: Writing - Original Draft, Visualization. Lei Wang: Data Curation, Funding acquisition. Yan Chen: Conceptualization, Formal analysis, Investigation, Resources, Writing - Original Draft, Writing - Review \& Editing, Supervision, Project administration, Funding acquisition. Hui Fang: Conceptualization, Formal analysis, Writing Original Draft, Writing - Review \& Editing, Visualization.

Your sincerely,

Corresponding Author: Yan Chen

On behalf of all co-authors

Affiliation: School of Medicine, University of Nottingham

E-mail: yan.chen.ac.uk@gmail.com 


\section{Statement for change of authorship}

In our revised version, we add Jiahui Wang and Cundian Yang to our author list because they have made significant contributions during the paper revision stage: 1) Jiahui Wang conducts and reports the additional experiment for security analysis. In addition, Jiahui Wang also reviews and edits this revised paper. 2) Cundian Yang helps Yuying Sun to conduct the other experiments including Ablation study (Section 4.3) and Comparisons with other state-of-theart zero-watermarking schemes (Section 4.4) for our revised paper.

All the co-authors have known and agreed with the addition of Jiahui Wang and Cundian Yang to our author list with their signatures as below.

\section{Signatures:}

Xiyao Liu

xiyao $\operatorname{Lin}$

Yuying Sun

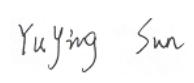

Jiahui Wang

Jia hui Wang

Cundian Yang

Cundian Yang

Yayun Zhang

Yayun Zhang

Lei Wang

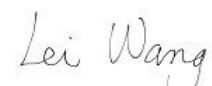

Yan Chen

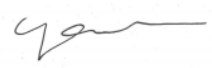

Hui Fang

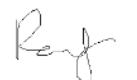

Your sincerely,

Corresponding Author: Yan Chen

On behalf of all co-authors

Affiliation: School of Medicine, University of Nottingham

E-mail: yan.chen.ac.uk@gmail.com 